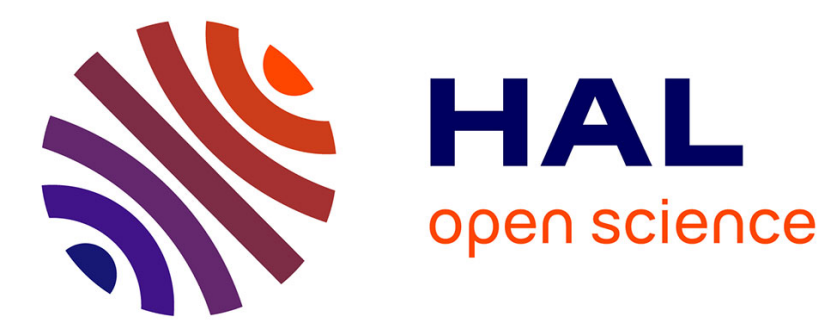

\title{
Identification of Bayesian posteriors for coefficients of chaos expansions
}

\author{
M. Arnst, R. Ghanem, Christian Soize
}

\section{To cite this version:}

M. Arnst, R. Ghanem, Christian Soize. Identification of Bayesian posteriors for coefficients of chaos expansions. Journal of Computational Physics, 2010, 229 (9), pp.3134-3154. 10.1016/j.jcp.2009.12.033 . hal-00684317

\section{HAL Id: hal-00684317 \\ https://hal.science/hal-00684317}

Submitted on 1 Apr 2012

HAL is a multi-disciplinary open access archive for the deposit and dissemination of scientific research documents, whether they are published or not. The documents may come from teaching and research institutions in France or abroad, or from public or private research centers.
L'archive ouverte pluridisciplinaire HAL, est destinée au dépôt et à la diffusion de documents scientifiques de niveau recherche, publiés ou non, émanant des établissements d'enseignement et de recherche français ou étrangers, des laboratoires publics ou privés. 


\title{
Identification of Bayesian posteriors for coefficients of chaos expansions
}

\author{
M. Arnst*a, R. Ghanem ${ }^{a}$, C. Soize ${ }^{b}$ \\ a210 KAP Hall, University of Southern California, Los Angeles, CA 90089, USA \\ ${ }^{\mathrm{b}}$ Université Paris-Est, Laboratoire de Modélisation et Simulation Multi-Échelle, \\ MSME FRE3160 CNRS, 5 bd Descartes, Champs-sur-Marne, 77454 \\ Marne-la-Vallée, Cedex 2, France
}

\begin{abstract}
This article is concerned with the identification of stochastic representations of random variables and fields from experimental data. The data used for the identification consist of measurements of several realizations of the uncertain quantities that must be represented. The random variables and fields are approximated by a polynomial chaos expansion, and the coefficients of this expansion are viewed as unknown parameters to be identified. It is shown how the Bayesian paradigm can be applied to formulate and solve the inverse problem. The estimated polynomial chaos coefficients are hereby themselves characterized as random variables whose probability density function is the Bayesian posterior. This allows to quantify the impact of missing experimental information on the accuracy of the identified coefficients, as well as of subsequent predictions. An illustration in stochastic aeroelastic stability analysis is provided to demonstrate the proposed methodology.
\end{abstract}

Key words: stochastic inversion, identification, Bayesian, maximum likelihood, polynomial chaos, validation, uncertainty quantification 


\section{Introduction}

Validation is a critical requirement for physics-based prediction models to find useful applications in prognosis and engineering design. The validation of a model involves providing a quantitative description of the proximity between the model and the reality that it purports to represent, with the objective of establishing the trustworthiness of the predictions. Stochastic approaches to model validation aim at using stochastic techniques to quantify the impact of missing data, modeling errors, and discretization errors on predictions.

Several approaches for constructing stochastic models have been proposed in the literature, see e.g. the reviews [1-4]. Parametric stochastic models accommodate uncertainty by modeling local physical features of a model, such as its geometrical parameters, fields of material properties and boundary conditions, by random variables and/or fields, see e.g. [1, 5]. Non-parametric stochastic models incorporate uncertainty by modeling global features of a model by random variables. An example is the non-parametric approach proposed by Soize $[6,7]$, where reduced matrix models are defined in terms of random matrices.

A key task in the construction of a stochastic model is the choice of the probability distribution of the random variables, fields, or matrices. A frequently adopted approach consists in choosing one of the "labeled" probability distributions, such as the Gaussian, lognormal or Wishart distribution, and in identifying the parameters of that distribution from experimental data. An example is the modeling of a scalar uncertain quantity by a Gaussian random variable, and the estimation of its mean and variance from experimental data. An alternative approach has its roots in

Email address: arnst@usc.edu (M. Arnst*). 
functional analysis, and consists in representing random variables or fields by a Polynomial Chaos Expansion (PCE), see e.g. [5, 8-11], and in identifying the coefficients of this expansion from experimental data. The latter approach is more versatile, in that it allows to represent a wider class of random variables, and also permits fast and efficient sampling from the identified random variables or fields.

Several methodologies for the identification of representations of random variables and fields from experimental data have already been proposed. They mostly rely on procedures from the theory of mathematical statistics, see e.g. [12, 13], such as the method of moments [14, 15], maximum likelihood [15-19], Bayesian inference [2022], maximum entropy [23] and minimum relative entropy [14, 16].

A significant challenge associated with these identification approaches consists in faithfully capturing the weight of available experimental data, and in developing error analysis capabilities to determine the value of additional data. In this context, the main objective of this article is to develop a systhematic methodology for quantifying the impact of missing experimental information on the accuracy of inferred PCEs representing random variables or fields. Ghanem and Doostan [24] and Ghanem et al. [18] have already proposed a methodology that relies on asymptotic properties of the identification method (as more and more data become available) to characterize uncertainties associated with limited data. However, many data sets encountered in practice are rather small to invoke asymptotic properties. We complement the previous work by developing an alternative methodology that relies on the Bayesian paradigm and is suitable for both small and large data sets.

This article is organized as follows. First, Secs. 2 and 3 recall the representation of random variables and fields using the Karhunen-Loeve and polynomial chaos expansions. Then, Sec. 4 summarizes the specific setting in which the methodology 
will be developed together with a concise statement of the task to be undertaken. Subsequently, Secs. 5 to 8, which constitute the core of this article, expound on the Bayesian identification of PCEs. Section 9 provides details to assist the reader in implementing the framework. Finally, Sec. 10 provides an illustration in stochastic aeroelastic stability analysis to demonstrate the proposed methodology.

\subsection{Notations}

The following notations are frequently used in this article. Let $\mathbb{N}$ and $\mathbb{R}$ denote the sets of respectively integers and real scalars. Any vector $\boldsymbol{x}=\left(x_{1}, \ldots, x_{n}\right) \in \mathbb{R}^{n}$ is identified with the $(n \times 1)$ column matrix of its components. Let $\boldsymbol{x}, \boldsymbol{y} \in \mathbb{R}^{n}$ be two real vectors. Then, $\boldsymbol{x}^{\mathrm{T}} \boldsymbol{y}$ denotes the Euclidean inner product such that $\boldsymbol{x}^{\mathrm{T}} \boldsymbol{y}=\sum_{k=1}^{n} x_{k} y_{k}$, and $\|\boldsymbol{x}\|$ the Euclidean norm such that $\|\boldsymbol{x}\|=\sqrt{\boldsymbol{x}^{\mathrm{T}} \boldsymbol{x}}$.

Let $\mathrm{M}_{n}(\mathbb{R})$ be the space of square $(n \times n)$ matrices $\boldsymbol{X}$ whose entries $X_{k \ell}$ are in $\mathbb{R}$. Then, $\operatorname{tr}(\boldsymbol{X})$ denotes the trace of $\boldsymbol{X}$ such that $\operatorname{tr}(\boldsymbol{X})=\sum_{k=1}^{n} X_{k k}$, and $\boldsymbol{X}^{\mathrm{T}}$ the transpose of $\boldsymbol{X}$. The tensor product $\boldsymbol{x} \otimes \boldsymbol{y}$ of $\boldsymbol{x}, \boldsymbol{y} \in \mathbb{R}^{n}$ is represented by the matrix $\boldsymbol{x} \boldsymbol{y}^{\mathrm{T}} \in \mathrm{M}_{n}(\mathbb{R})$.

Throughout the article, $(\Omega, \mathcal{F}, P)$ is a probability measure space, where $\Omega$ is the sample space of outcomes, $\mathcal{F}$ the $\sigma$-algebra of events and $P: \mathcal{F} \rightarrow[0,1]$ the probability measure. The symbol $E\{\cdot\}$ denotes the integral with respect to the probability measure, i.e. the mathematical expectation. For instance, for a mapping $\varphi: \Omega \rightarrow \mathbb{R}$ :

$$
E\{\varphi\}=\int_{\Omega} \varphi(\omega) d P(\omega)
$$




\section{Representation of stochastic fields}

This section briefly recalls the construction of finite-dimensional approximations of stochastic fields by projection on Hilbertian bases. The reader is referred to [5] and references therein for more details.

\subsection{Projection on Hilbertian basis}

Consider a second-order and mean-square continuous stochastic field $\{\boldsymbol{a}(\boldsymbol{x}), \boldsymbol{x} \in \mathcal{D}\}$ defined on $(\Omega, \mathcal{F}, P)$, with values in $\mathbb{R}^{m}$, and indexed by a bounded closed set $\mathcal{D} \subset$ $\mathbb{R}^{n}$. Let $\left\{\boldsymbol{v}_{j}(\boldsymbol{x}), 1 \leq j \leq+\infty\right\}$ be a Hilbertian basis of functions from $\mathcal{D}$ into $\mathbb{R}^{m}$, that is to say a complete collection of orthonormal functions that satisfy

$$
\int_{\mathcal{D}} \boldsymbol{v}_{j}(\boldsymbol{x})^{\mathrm{T}} \boldsymbol{v}_{k}(\boldsymbol{x}) d \boldsymbol{x}=\delta_{j k}
$$

where $\delta_{j k}$ is the Kronecker symbol, equal to 1 if $j=k$, and to 0 otherwise. The stochastic field $\{\boldsymbol{a}(\boldsymbol{x}), \boldsymbol{x} \in \mathcal{D}\}$ can be represented on this Hilbertian basis as

$$
\boldsymbol{a}(\boldsymbol{x})=\sum_{j=1}^{+\infty} a_{j} \boldsymbol{v}_{j}(\boldsymbol{x})
$$

in which the random variables $a_{j}$ are defined on $(\Omega, \mathcal{F}, P)$, valued in $\mathbb{R}$, of the second order, and such that

$$
a_{j}=\int_{\mathcal{D}} \boldsymbol{a}(\boldsymbol{x})^{\mathrm{T}} \boldsymbol{v}_{j}(\boldsymbol{x}) d \boldsymbol{x} .
$$




\subsection{Karhunen-Loeve expansion}

The Karhunen-Loeve expansion is a projection of the stochastic field $\{\boldsymbol{a}(\boldsymbol{x}), \boldsymbol{x} \in \mathcal{D}\}$ onto a particular Hilbertian basis, which has the following form:

$$
\boldsymbol{a}(\boldsymbol{x})=\underline{\boldsymbol{a}}(\boldsymbol{x})+\sum_{j=1}^{+\infty} \sqrt{\lambda_{j}} \eta_{j} \boldsymbol{v}_{j}(\boldsymbol{x}),
$$

in which $\underline{\boldsymbol{a}}(\boldsymbol{x})=E\{\boldsymbol{a}(\boldsymbol{x})\}$ is the mean field, and the functions $\boldsymbol{v}_{j}(\boldsymbol{x})$ are the solutions to the eigenvalue problem

$$
\int_{\mathcal{D}} \boldsymbol{R}_{\boldsymbol{a}}\left(\boldsymbol{x}, \boldsymbol{x}^{\prime}\right) \boldsymbol{v}_{j}\left(\boldsymbol{x}^{\prime}\right) d \boldsymbol{x}^{\prime}=\lambda_{j} \boldsymbol{v}_{j}(\boldsymbol{x})
$$

normalized such that

$$
\int_{\mathcal{D}}\left\|\boldsymbol{v}_{j}(\boldsymbol{x})\right\|^{2} d \boldsymbol{x}=1
$$

where $\boldsymbol{R}_{\boldsymbol{a}}\left(\boldsymbol{x}, \boldsymbol{x}^{\prime}\right)=E\left\{(\boldsymbol{a}(\boldsymbol{x})-\underline{\boldsymbol{a}}(\boldsymbol{x})) \otimes\left(\boldsymbol{a}\left(\boldsymbol{x}^{\prime}\right)-\underline{\boldsymbol{a}}\left(\boldsymbol{x}^{\prime}\right)\right)\right\}$ is the matrix-valued covariance function of $\{\boldsymbol{a}(\boldsymbol{x}), \boldsymbol{x} \in \mathcal{D}\}$. It can be shown that:

(i) Due to the mean-square continuity of $\{\boldsymbol{a}(\boldsymbol{x}), \boldsymbol{x} \in \mathcal{D}\}$ and the boundedness of $\mathcal{D}$, the integral covariance operator in (6) is Hilbert-Schmidt. Due to the symmetry of its kernel, i.e. $\forall \boldsymbol{x}, \boldsymbol{x}^{\prime} \in \mathcal{D}: \boldsymbol{R}_{\boldsymbol{a}}\left(\boldsymbol{x}, \boldsymbol{x}^{\prime}\right)=\boldsymbol{R}_{\boldsymbol{a}}\left(\boldsymbol{x}, \boldsymbol{x}^{\prime}\right)^{\mathrm{T}}$, it is self-adjoint. Due to the positivity of the covariance matrix, i.e. $\forall \boldsymbol{x} \in \mathcal{D}: \boldsymbol{R}_{\boldsymbol{a}}(\boldsymbol{x}, \boldsymbol{x})$ is positive, it is, moreover, positive. Hence, the eigenfunctions $\boldsymbol{v}_{j}(\boldsymbol{x})$ form a complete orthonormal basis, the eigenvalues $\lambda_{j}$ form a decreasing sequence of positive values $\lambda_{1} \geq \ldots \geq$ $\lambda_{j} \geq \ldots \rightarrow 0$, and the series of eigenvalues is convergent:

$$
\int_{\mathcal{D}} E\left\{\|\boldsymbol{a}(\boldsymbol{x})\|^{2}\right\} d \boldsymbol{x}=\int_{\mathcal{D}} \operatorname{tr}\left(\boldsymbol{R}_{\boldsymbol{a}}(\boldsymbol{x}, \boldsymbol{x})\right) d \boldsymbol{x}=\sum_{j=1}^{+\infty} \lambda_{j}<+\infty
$$

(ii) The random variables $\eta_{j}$ are of the second-order, centered, and orthonormal (how- 
ever, they are not, in general, independent):

$$
\begin{array}{ll}
\forall j: & E\left\{\eta_{j}\right\}=0, \\
\forall j, k: & E\left\{\eta_{j} \eta_{k}\right\}=\delta_{j k},
\end{array}
$$

(iii) Representation (5) converges in the mean square sense, as well as uniformly.

Truncating expansion (5) after the $d$-th term results in the following approximation of the stochastic field:

$$
\boldsymbol{a}^{\mathrm{d}}(\boldsymbol{x})=\underline{\boldsymbol{a}}(\boldsymbol{x})+\sum_{j=1}^{d} \sqrt{\lambda_{j}} \eta_{j} \boldsymbol{v}_{j}(\boldsymbol{x}),
$$

in which $\boldsymbol{\eta}$ is a random variable defined on $(\Omega, \mathcal{F}, P)$ with values in $\mathbb{R}^{d}$, whose components are the random variables $\eta_{j}$. The truncation error reads as

$$
\boldsymbol{a}(\boldsymbol{x})-\boldsymbol{a}^{\mathrm{d}}(\boldsymbol{x})=\sum_{j=d+1}^{+\infty} \sqrt{\lambda_{j}} \eta_{j} \boldsymbol{v}_{j}(\boldsymbol{x}) .
$$

Based upon (7) and (10), the following estimate of the magnitude of the truncation error is obtained:

$$
\int_{\mathcal{D}} E\left\{\left\|\boldsymbol{a}(\boldsymbol{x})-\boldsymbol{a}^{\mathrm{d}}(\boldsymbol{x})\right\|^{2}\right\} d \boldsymbol{x}=\sum_{j=d+1}^{+\infty} \lambda_{j} .
$$

The Karhunen-Loeve expansion can be shown to be optimal, in the sense that basis functions satisfying (6) minimize the magnitude of the truncation error.

\section{Polynomial chaos expansion}

The discretization of random variables by projection on polynomial chaos is recalled next. The PCE provides for a second-order random variable $\boldsymbol{a}$ defined on $(\Omega, \mathcal{F}, P)$ with values in the finite dimensional space $\mathbb{R}^{m}$ a representation as

$$
\boldsymbol{a}=\sum_{\boldsymbol{\alpha},|\boldsymbol{\alpha}|=0}^{+\infty} \boldsymbol{p}_{\boldsymbol{\alpha}} H_{\boldsymbol{\alpha}}(\boldsymbol{\xi})
$$


in which $\boldsymbol{\alpha}=\left(\alpha_{1}, \ldots, \alpha_{d}\right) \in \mathbb{N}^{m}$ is a multi-index with modulus $|\boldsymbol{\alpha}|=\alpha_{1}+\ldots+\alpha_{m}$, each $\boldsymbol{p}_{\boldsymbol{\alpha}}$ a vector in $\mathbb{R}^{m}, \boldsymbol{\xi}$ a second-order random variable defined on $(\Omega, \mathcal{F}, P)$ with values in $\mathbb{R}^{m}$, and the functions $H_{\boldsymbol{\alpha}}$ form a complete set of orthonormal functions that satisfy

$$
E\left\{H_{\boldsymbol{\alpha}}(\boldsymbol{\xi}) H_{\boldsymbol{\beta}}(\boldsymbol{\xi})\right\}=\delta_{\boldsymbol{\alpha} \boldsymbol{\beta}}=\delta_{\alpha_{1} \beta_{1}} \times \ldots \times \delta_{\alpha_{m} \beta_{m}} .
$$

The random variable $\boldsymbol{\xi}$ is often chosen to have independent components with either a Gaussian, uniform, gamma, Chebyshev or beta probability distribution. The function $H_{\boldsymbol{\alpha}}$ is then a multi-dimensional polynomial of the form $H_{\boldsymbol{\alpha}}(\boldsymbol{\xi})=h_{\alpha_{1}}\left(\xi_{1}\right) \times$ $\ldots \times h_{\alpha_{m}}\left(\xi_{m}\right)$, in which $h_{\alpha_{j}}$ is respectively the one-dimensional normalized Hermite, Legendre, Laguerre, Chebyshev or Jacobi polynomial of order $\alpha_{j}$. The extension to the case of basic random variables with arbitrary probability distribution has been completed in [8].

If random variable $\boldsymbol{a}$ is valued in a finite dimensional space, as we have assumed, then representation (14) converges in the mean-square sense. However, it should be stressed that, in the present state of the art in mathematics, the convergence of a chaos expansion for a second-order random variable with values in an infinitedimensional space can be obtained only if the germ $\boldsymbol{\xi}$ is Gaussian [25].

Truncating expansion (14) after polynomials of order $r$ results in the following approximation of $\boldsymbol{a}$ :

$$
\boldsymbol{a}^{\mathrm{r}}=\sum_{\boldsymbol{\alpha},|\boldsymbol{\alpha}|=0}^{r} \boldsymbol{p}_{\boldsymbol{\alpha}} H_{\boldsymbol{\alpha}}(\boldsymbol{\xi})
$$

incurring the following truncation error:

$$
\boldsymbol{a}-\boldsymbol{a}^{\mathrm{r}}=\sum_{\boldsymbol{\alpha},|\boldsymbol{\alpha}|=r+1}^{+\infty} \boldsymbol{p}_{\boldsymbol{\alpha}} H_{\boldsymbol{\alpha}}(\boldsymbol{\xi}) .
$$

Equation (15) enables the following estimate of its magnitude:

$$
E\left\{\left\|\boldsymbol{a}-\boldsymbol{a}^{\mathrm{r}}\right\|^{2}\right\}=E\left\{\|\boldsymbol{a}\|^{2}\right\}-\sum_{\boldsymbol{\alpha},|\boldsymbol{\alpha}|=0}^{r}\left\|\boldsymbol{p}_{\boldsymbol{\alpha}}\right\|^{2} .
$$


It is noted that the number of terms in expansion (16) is equal to

$$
\left|\left\{\boldsymbol{\alpha} \in \mathbb{N}^{m}, 0 \leq|\boldsymbol{\alpha}| \leq r\right\}\right|=\sum_{j=0}^{r} \frac{(j+m-1) !}{j !(m-1) !}
$$

\section{Problem setting}

It is assumed that a complex natural or engineered system is under study. The behavior of this system is assumed to exhibit variability, in the sense that each time the system is polled to measure some quantity of interest, a different value is obtained, as if it were sampled from a probability distribution. Let a stochastic model be built of the behavior of the system under study. Let this stochastic model accommodate variability by modeling its fields of material properties, geometrical characteristics and/or boundary conditions by random variables or fields. We are interested here in the identification of a probabilistic characterization of these random variables or fields from experimental data.

We would like to point out that the present setting is quite different from the context addressed in references [20-22]. The latter contributions deal with the characterization of a system that is essentially perceived as deterministic. The objective of the inverse problems considered in those works lies in the description of that system in terms of ideally deterministic quantities, such as applied loadings or fields of material properties. Bayesian inference techniques are applied for the purpose of regularization, and involve the quantification of the uncertainty in the identified quantities stemming from experimental noise or missing data. In contrast, the present paper deals with the characterization of a system that is explicitly perceived as stochastic. The objective of the inverse problem lies, here, in the description of this system in terms of stochastic quantities that are representative of the system variability. 


\section{Data available for the identification}

It is assumed that a probabilistic characterization of a finite number, say $m$, of uncertain quantities needs to be identified. These can, for instance, be $m$ scalar material properties, geometrical characteristics or applied loadings. Or, they can also be the values taken by fields of material properties, geometrical characteristics or applied loadings, at $m$ prescribed locations, or the projections of such fields on $m$ appropriate basis functions (cfr. Sec. 2).

It is assumed that a data set

$$
\left\{\boldsymbol{a}^{(j)}, 1 \leq j \leq n\right\}
$$

of $n$ independent and identically-distributed realizations of the uncertain quantities has been observed, each realization $\boldsymbol{a}^{(j)}$ valued in $\mathbb{R}^{m}$.

\section{Maximum likelihood stochastic inversion}

This and the next section are concerned with the identification, from the data, of a probabilistic characterization of the uncertain quantities in terms of a random variable $\boldsymbol{a}$ defined on $(\Omega, \mathcal{F}, P)$ with values in $\mathbb{R}^{m}$. As an ingredient for the Bayesian inverse method that will be elaborated in the next section, we address in this section the maximum likelihood identification of $\boldsymbol{a}$.

\subsection{Discretization using a polynomial chaos expansion}

Since $\boldsymbol{a}$ is a mapping from $\Omega$ into $\mathbb{R}^{m}$, its identification constitutes a functional, hence infinite-dimensional, inverse problem. It can be discretized by approximating $\boldsymbol{a}$ by 
a truncated PCE of dimension $m$ and order $r$ of the form

$$
\boldsymbol{a}^{\mathrm{r}}(\boldsymbol{p})=\sum_{\boldsymbol{\alpha},|\boldsymbol{\alpha}|=0}^{r} \boldsymbol{p}_{\boldsymbol{\alpha}} H_{\boldsymbol{\alpha}}(\boldsymbol{\xi})
$$

and then viewing the coefficients $\boldsymbol{p}=\left\{\boldsymbol{p}_{\boldsymbol{\alpha}}, 0 \leq|\boldsymbol{\alpha}|<r\right\}$ of this expansion as unknown parameters that must be estimated.

It is emphasized that, here, the germ $\boldsymbol{\xi}$ is taken as a random variable with values in $\mathbb{R}^{m}$, i.e. the dimension of the space of values of $\boldsymbol{\xi}$ is taken equal to the dimension of the space of values of $\boldsymbol{a}$.

\subsection{Identification of a polynomial chaos expansion}

The method of maximum likelihood $[12,13]$ can readily be applied to the identification of $\boldsymbol{p}$. The likelihood of $\boldsymbol{p}$ given the data is defined as

$$
L(\boldsymbol{p})=\prod_{j=1}^{n} f_{\boldsymbol{a}}\left(\boldsymbol{a}^{(j)} \mid \boldsymbol{p}\right)
$$

in which $f_{\boldsymbol{a}}(\cdot \mid \boldsymbol{p})$ denotes the Probability Density Function (PDF) of $\boldsymbol{a}^{\mathrm{r}}(\boldsymbol{p})$, which depends on $\boldsymbol{p}$. The method of maximum likelihood involves optimizing $L(\boldsymbol{p})$ :

$$
\hat{\boldsymbol{p}}=\arg \max _{\boldsymbol{p}} L(\boldsymbol{p}) .
$$

In other words, parameters are chosen for which the data are most likely.

\subsection{Discretization using a reduced representation}

A disadvantage of expansion (21) is that the dimension of $\boldsymbol{p}$, that is to say the number of scalar parameters to be estimated, increases quickly with $m$, cfr. (19). Unfortunately, the computational cost associated with (23) increases quickly with 
the dimension of $\boldsymbol{p}$. A methodology for the identification of representations of reduced dimension is therefore presented next.

With reference to (11), consider an approximation of $\boldsymbol{a}$ of the form:

$$
\boldsymbol{a}^{\mathrm{d}}\left(\boldsymbol{p}^{0}\right)=\boldsymbol{p}^{0}+\sum_{j=1}^{d} \sqrt{\hat{\lambda}_{j}} \eta_{j} \hat{\boldsymbol{v}}_{j}
$$

in which $d$ is the reduced dimension, with $1 \leq d \leq m$, and $\hat{\lambda}_{j}$ and $\hat{\boldsymbol{v}}_{j}$ the $d$ dominant eigenvalues and eigenvectors of the sample covariance matrix $\hat{\boldsymbol{C}}_{\boldsymbol{a}}$ :

$$
\begin{aligned}
& \hat{\boldsymbol{m}}_{\boldsymbol{a}}=\frac{1}{n} \sum_{j=1}^{n} \boldsymbol{a}^{(j)} \\
& \hat{\boldsymbol{C}}_{\boldsymbol{a}}=\frac{1}{n-1} \sum_{j=1}^{n}\left(\boldsymbol{a}^{(j)}-\hat{\boldsymbol{m}}_{\boldsymbol{a}}\right) \otimes\left(\boldsymbol{a}^{(j)}-\hat{\boldsymbol{m}}_{\boldsymbol{a}}\right)
\end{aligned}
$$

Let the random variables $\eta_{j}$ in (24) be collected in a random vector $\boldsymbol{\eta}$ with values in $\mathbb{R}^{d}$. Let $\boldsymbol{\eta}$ be approximated by a PCE of dimension $d$ and order $r$ :

$$
\boldsymbol{\eta}^{\mathrm{r}}\left(\boldsymbol{p}^{\delta}\right)=\sum_{\boldsymbol{\alpha},|\boldsymbol{\alpha}|=1}^{r} \boldsymbol{p}_{\boldsymbol{\alpha}}^{\delta} H_{\boldsymbol{\alpha}}(\boldsymbol{\xi})
$$

Upon injecting (27) in (24), the following reduced representation is then obtained:

$$
\boldsymbol{a}^{\mathrm{d}, \mathrm{r}}(\boldsymbol{p})=\boldsymbol{p}^{0}+\sum_{j=1}^{d} \sqrt{\hat{\lambda}_{j}} \sum_{\boldsymbol{\alpha},|\boldsymbol{\alpha}|=1}^{r} p_{\boldsymbol{\alpha} j}^{\delta} H_{\boldsymbol{\alpha}}(\boldsymbol{\xi}) \hat{\boldsymbol{v}}_{j} .
$$

The parameters $\boldsymbol{p}=\left\{\boldsymbol{p}^{0}, \boldsymbol{p}^{\delta}\right\}$ are, this time, viewed as unknown parameters that must be estimated from the data. They comprise the mean $\boldsymbol{p}^{0}$, as well as the coefficients $\boldsymbol{p}^{\delta}=\left\{\boldsymbol{p}_{\boldsymbol{\alpha}}^{\delta}, 1 \leq|\boldsymbol{\alpha}| \leq r\right\}$ that control the fluctuating part of the expansion.

It is emphasized that the germ $\boldsymbol{\xi}$ is valued in $\mathbb{R}^{d}$, with $d<m$ usually. In other words, the dimension $d$ of the space of values of $\boldsymbol{\xi}$ is, this time, smaller than the dimension $m$ of the space of values of $\boldsymbol{a}$.

It is crucial to choose $d$ sufficiently large so as to limit the accuracy loss that may occur due to the dimension reduction below an admissible tolerance. We recommend 
estimating the required dimension from the eigenvalue structure of $\hat{\boldsymbol{C}}_{\boldsymbol{a}}$, namely choosing $d$ such that the following equation is satisfied for sufficiently small $\epsilon$ :

$$
\sum_{j=d+1}^{m} \hat{\lambda}_{j}=\epsilon \sum_{j=1}^{m} \hat{\lambda}_{j}
$$

In addition, convergence analyses can be performed to ensure that a sufficient number of dimensions is retained.

\subsection{Identification of a reduced representation}

The method of maximum likelihood cannot readily be applied to the identification of $\boldsymbol{p}^{0}$ and $\boldsymbol{p}^{\delta}$ due to the following difficulty. Equation (28) defines the random variable $\boldsymbol{a}^{\mathrm{d}, \mathrm{r}}(\boldsymbol{p})$ with values in $\mathbb{R}^{m}$ as the transformation through a parameterized deterministic mapping of a random variable $\boldsymbol{\xi}$ with values in $\mathbb{R}^{d}$, with $d<m$ usually. For fixed $\boldsymbol{p}$, the values taken by $\boldsymbol{a}^{\mathrm{d}, \mathrm{r}}(\boldsymbol{p})$ therefore lie on a $d$-dimensional hypersurface in $\mathbb{R}^{m}$, and the PDF $f_{\boldsymbol{a}}(\cdot \mid \boldsymbol{p})$ vanishes everywhere in $\mathbb{R}^{m}$, except on this hypersurface. The shape of this hypersurface depends on $\boldsymbol{p}$. However, for a reasonably high number $n$ of observations and low order $r$ of the PCE, it will generally be impossible to find parameters $\boldsymbol{p}$ such that all observations $\boldsymbol{a}^{(j)}$, being a priori arbitrary vectors in $\mathbb{R}^{m}$, lie on the hypersurface. In other words, it will generally be impossible to find parameters $\boldsymbol{p}$ such that all $\boldsymbol{a}^{(j)}$ belong to the support of $f_{\boldsymbol{a}}(\cdot \mid \boldsymbol{p})$. The likelihood

$$
L(\boldsymbol{p})=\prod_{j=1}^{n} f_{\boldsymbol{a}}\left(\boldsymbol{a}^{(j)} \mid \boldsymbol{p}\right),
$$

in which $f_{\boldsymbol{a}}(\cdot \mid \boldsymbol{p})$ denotes the PDF of $\boldsymbol{a}^{\mathrm{d}, \mathrm{r}}(\boldsymbol{p})$, therefore generally vanishes for all $\boldsymbol{p}$, which renders the optimization problem

$$
\hat{\boldsymbol{p}}=\arg \max _{\boldsymbol{p}} L(\boldsymbol{p})
$$

meaningless. 
We propose a two-step identification procedure to overcome this difficulty. First, we propose to identify $\boldsymbol{p}^{\delta}$ by invoking the maximum likelihood principle in terms of the reduced coordinates of the representation. The likelihood of $\boldsymbol{p}^{\delta}$ is thus defined by

$$
L^{\delta}\left(\boldsymbol{p}^{\delta}\right)=\prod_{j=1}^{n} f_{\boldsymbol{\eta}}\left(\boldsymbol{\eta}^{(j)} \mid \boldsymbol{p}^{\delta}\right)
$$

in which $f_{\boldsymbol{\eta}}\left(\cdot \mid \boldsymbol{p}^{\delta}\right)$ denotes the PDF of $\boldsymbol{\eta}^{\mathrm{r}}\left(\boldsymbol{p}^{\delta}\right)$, and the vectors $\boldsymbol{\eta}^{(j)}$ are the projection of the fluctuating part of the observations onto the reduction basis:

$$
\eta_{k}^{(j)}=\frac{\left(\boldsymbol{a}^{(j)}-\hat{\boldsymbol{m}}_{\boldsymbol{a}}\right)^{\mathrm{T}} \hat{\boldsymbol{v}}_{k}}{\sqrt{\hat{\lambda}_{k}}} .
$$

The parameters maximizing the likelihood are then selected:

$$
\hat{\boldsymbol{p}}^{\delta}=\arg \max _{\boldsymbol{p}^{\delta}} L^{\delta}\left(\boldsymbol{p}^{\delta}\right)
$$

The abovementioned difficulty is, in this way, avoided, since the germ $\boldsymbol{\xi}$ in (27), as well as the vectors $\boldsymbol{\eta}^{(j)}$ defined by (33), take their values in a $d$-dimensional space.

Subsequently, we propose to identify $\boldsymbol{p}^{0}$ by invoking the maximum likelihood principle using the first-order marginal PDFs $f_{a_{k}}\left(\cdot \mid \boldsymbol{p}^{0}, \hat{\boldsymbol{p}}^{\delta}\right)$ of the components $a_{k}^{\mathrm{d}, \mathrm{r}}\left(\boldsymbol{p}^{0}, \hat{\boldsymbol{p}}^{\delta}\right)$ of $\boldsymbol{a}^{\mathrm{d}, \mathrm{r}}\left(\boldsymbol{p}^{0}, \hat{\boldsymbol{p}}^{\delta}\right)$. The likelihood of $\boldsymbol{p}^{0}$ is thus defined by

$$
L^{0}\left(\boldsymbol{p}^{0}\right)=\prod_{k=1}^{m} f_{a_{k}}\left(a_{k}^{(j)} \mid \boldsymbol{p}^{0}, \hat{\boldsymbol{p}}^{\delta}\right)
$$

The parameters maximizing the likelihood are then selected:

$$
\hat{\boldsymbol{p}}^{0}=\arg \max _{\boldsymbol{p}^{0}} L^{0}\left(\boldsymbol{p}^{0}\right)
$$

The abovementioned difficulty is avoided by using only the first-order marginal PDFs since the dimension of the space of values of the germ $\boldsymbol{\xi}$ is at least 1 . 


\section{Bayesian stochastic inversion}

A perfect probabilistic characterization of uncertain quantities cannot, in general, be deduced from a data set of only a finite number of realizations. It is shown next how the Bayesian method, see e.g. [26-29], can be applied to quantify the incomplete knowledge of $\boldsymbol{a}$ due to the finite length of the data set.

\subsection{Identification of a polynomial chaos expansion}

The Bayesian method can readily be applied to the identification of the parameters $\boldsymbol{p}$ of the PCE approximation (21) of $\boldsymbol{a}$. The Bayesian approach uses PDFs to represent available information on imperfectly known parameters. First, a prior PDF $\rho(\boldsymbol{p})$ is constructed representing whichever information is available on $\boldsymbol{p}$ in advance of acquiring the data. Then, the posterior PDF $\sigma(\boldsymbol{p})$, representing all information available after making the observations, is obtained as follows:

$$
\begin{aligned}
\sigma(\boldsymbol{p}) & =c \rho(\boldsymbol{p}) L(\boldsymbol{p}) \\
& =c \rho(\boldsymbol{p}) \prod_{j=1}^{n} f_{\boldsymbol{a}}\left(\boldsymbol{a}^{(j)} \mid \boldsymbol{p}\right),
\end{aligned}
$$

in which $c$ is a normalization constant, $L(\boldsymbol{p})$ still denotes the likelihood of $\boldsymbol{p}$, and $f_{\boldsymbol{a}}(\cdot \mid \boldsymbol{p})$ still denotes the PDF of $\boldsymbol{a}^{\mathrm{r}}(\boldsymbol{p})$.

\subsection{Identification of a reduced representation}

The Bayesian method can be applied as follows to the identification of the parameters $\boldsymbol{p}^{0}$ and $\boldsymbol{p}^{\delta}$ of the reduced approximation (28) of $\boldsymbol{a}$. Let the PDF $\rho\left(\boldsymbol{p}^{\delta}\right)$ represent 
the prior information available on $\boldsymbol{p}^{\delta}$. The posterior on $\boldsymbol{p}^{\delta}$ then reads as

$$
\sigma\left(\boldsymbol{p}^{\delta}\right)=c^{\delta} \rho\left(\boldsymbol{p}^{\delta}\right) \prod_{j=1}^{n} f_{\boldsymbol{\eta}}\left(\boldsymbol{\eta}^{(j)} \mid \boldsymbol{p}^{\delta}\right)
$$

in which $c^{\delta}$ is a normalization constant, $f_{\boldsymbol{\eta}}\left(\cdot \mid \boldsymbol{p}^{\delta}\right)$ still denotes the PDF of $\boldsymbol{\eta}^{\mathrm{r}}\left(\boldsymbol{p}^{\delta}\right)$, and each $\boldsymbol{\eta}^{(j)}$ is still defined by (33).

Similarly, letting PDF $\rho\left(\boldsymbol{p}^{0}\right)$ represent the prior information available on $\boldsymbol{p}^{0}$, the posterior on $\boldsymbol{p}^{0}$ reads as

$$
\sigma\left(\boldsymbol{p}^{0} \mid \boldsymbol{p}^{\delta}\right)=c^{0}\left(\boldsymbol{p}^{\delta}\right) \rho\left(\boldsymbol{p}^{0}\right) \prod_{j=1}^{n} \prod_{k=1}^{m} f_{a_{k}}\left(a_{k}^{(j)} \mid \boldsymbol{p}^{0}, \boldsymbol{p}^{\delta}\right)
$$

in which $c^{0}\left(\boldsymbol{p}^{\delta}\right)$ is a normalization constant, and $f_{a_{k}}\left(\cdot \mid \boldsymbol{p}^{0}, \boldsymbol{p}^{\delta}\right)$ the PDF of $a_{k}^{\mathrm{d}, \mathrm{r}}\left(\boldsymbol{p}^{0}, \boldsymbol{p}^{\delta}\right)$.

Finally, the posterior on $\boldsymbol{p}=\left\{\boldsymbol{p}^{0}, \boldsymbol{p}^{\delta}\right\}$ is obtained as

$$
\begin{aligned}
\sigma(\boldsymbol{p}) & =\sigma\left(\boldsymbol{p}^{0} \mid \boldsymbol{p}^{\delta}\right) \sigma\left(\boldsymbol{p}^{\delta}\right) \\
& =c^{0}\left(\boldsymbol{p}^{\delta}\right) c^{\delta} \rho\left(\boldsymbol{p}^{0}\right) \rho\left(\boldsymbol{p}^{\delta}\right) \prod_{j=1}^{n} \prod_{k=1}^{m} f_{a_{k}}\left(a_{k}^{(j)} \mid \boldsymbol{p}^{0}, \boldsymbol{p}^{\delta}\right) \prod_{j=1}^{n} f_{\boldsymbol{\eta}}\left(\boldsymbol{\eta}^{(j)} \mid \boldsymbol{p}^{\delta}\right) .
\end{aligned}
$$

It should be noted that the posterior (38) on $\boldsymbol{p}^{\delta}$ is independent of $\boldsymbol{p}^{0}$. In contrast, the posterior (39) on $\boldsymbol{p}^{0}$ depends on $\boldsymbol{p}^{\delta}$ since the likelihood (35) of $\boldsymbol{p}^{0}$ depends on $\boldsymbol{p}^{\delta}$.

\subsection{Selection of the prior PDF}

Gaussian [22], noninformative [26], conjugate [27], reference [28], and maximum entropy [29] priors have been proposed and used in the literature. We recommend using the improper uniform PDF as a noninformative prior on the polynomial coefficients if no information is available concerning these coefficients in advance of observing the data. And we suggest using a maximum entropy prior when information concerning their mean, covariance or other generalized moments is available. A maximum entropy prior can be constructed following e.g. the approach by [30]. 


\subsection{Remark concerning uncertainty in the covariance estimate}

It should be noted that, while the random variables $\eta_{j}$ in the Karhunen-Loeve expansion (5) are orthonormal, neither our maximum likelihood procedure (Sec. 6.4), nor our Bayesian procedure (Sec. 7.2) enforces the random variables $\eta_{j}$ in (24) to be orthonormal. In other words, the coefficients $\boldsymbol{p}_{\boldsymbol{\alpha}}^{\delta}$ in (27) are not required to be such that random vector $\boldsymbol{\eta}^{\mathrm{r}}\left(\boldsymbol{p}^{\delta}\right)$ in (27) has orthonormal components.

The reason is the following. Due to the finite length of the data set, the sample covariance matrix $\hat{\boldsymbol{C}}_{\boldsymbol{a}}$ is only an approximation of the covariance matrix that would ideally represent the covariance of the uncertain quantities to be characterized. The scalars $\hat{\lambda}_{j}$ and the vectors $\hat{\boldsymbol{v}}_{j}$ in (24) are chosen as the eigenvalues and eigenvectors of $\hat{\boldsymbol{C}}_{\boldsymbol{a}}$. Hence, if we had required the random variables $\eta_{j}$ in (24) to be orthonormal, then we would effectively have enforced $\hat{\boldsymbol{C}}_{\boldsymbol{a}}$ to be the covariance matrix of $\boldsymbol{a}$ (up to a small discrepancy due to the dimension reduction), and we would not have accommodated in the posterior PDF (40) the uncertainty in the covariance due to the finite length of the data set. By not enforcing orthonormality, we allow the Bayesian machinery to accommodate the aforementioned uncertainty in the posterior.

\section{Polynomial chaos expansion with random coefficients}

The uncertainty in the coefficients of the representation of $\boldsymbol{a}$, which is represented by the Bayesian posterior, can explicitly be accommodated in this representation by modeling these coefficients themselves by random variables, see e.g $[9,18,24,31]$.

To be specific, let the methodology outlined in Section 7.1 have been followed to 
approximate the random variable $\boldsymbol{a}$ by a PCE of the form

$$
\boldsymbol{a}^{\mathrm{r}}(\boldsymbol{p})=\sum_{\boldsymbol{\alpha},|\boldsymbol{\alpha}|=0}^{r} \boldsymbol{p}_{\boldsymbol{\alpha}} H_{\boldsymbol{\alpha}}\left(\xi_{1}, \ldots, \xi_{m}\right)
$$

and obtain a posterior $\sigma(\boldsymbol{p})$ of form (37). Let $\boldsymbol{p}$ collect $\tilde{m}$ scalar parameters, with

$$
\tilde{m}=m \sum_{j=0}^{p} \frac{(j+m-1) !}{j !(m-1) !} .
$$

Consider then the PCE of dimension $\tilde{m}$ and order $\tilde{r}$ of the form

$$
\boldsymbol{p}^{\tilde{r}}=\sum_{\boldsymbol{\beta},|\boldsymbol{\beta}|=0}^{\tilde{r}} \boldsymbol{q}_{\boldsymbol{\beta}} H_{\boldsymbol{\beta}}\left(\xi_{m+1}, \ldots, \xi_{m+\tilde{m}}\right)
$$

where the the coefficients $\boldsymbol{q}=\left\{\boldsymbol{q}_{\boldsymbol{\beta}}, 0 \leq|\boldsymbol{\beta}| \leq \tilde{r}\right\}$ and the order $\tilde{r}$ are such that the PDF of $\boldsymbol{p}^{\tilde{r}}$ is a sufficiently accurate approximation of $\sigma(\boldsymbol{p})$ on $\mathbb{R}^{\tilde{m}}$.

The random variables $\left\{\xi_{j}, m+1 \leq j \leq m+\tilde{m}\right\}$ in PCE (43) are independent of the random variables $\left\{\xi_{j}, 1 \leq j \leq m\right\}$ in $\mathrm{PCE}(41)$ to reflect the fact that the variability in the observables, which is a property of the system under study, is independent of the incomplete knowledge of this variability, which is a property of the analyst.

Upon introducing (43) in (41), a random variable

$$
\boldsymbol{a}^{\mathrm{r}, \tilde{r}}=\sum_{\boldsymbol{\alpha},|\boldsymbol{\alpha}|=0}^{r} \sum_{\boldsymbol{\beta},|\boldsymbol{\beta}|=0}^{\tilde{r}} \boldsymbol{q}_{\boldsymbol{\beta} \boldsymbol{\alpha}} H_{\boldsymbol{\beta}}\left(\xi_{m+1}, \ldots, \xi_{m+\tilde{m}}\right) H_{\boldsymbol{\alpha}}\left(\xi_{1}, \ldots, \xi_{m}\right)
$$

is obtained, which represents the variability in the system under study, as well as the incomplete knowledge of this variability.

This reasoning can readily be extended to the reduced representations of the form (28), and this extension is therefore not explicitly further elaborated here. 


\section{Implementation}

Algorithm 1 lists step by step how the likelihood $L(\boldsymbol{p})$ defined by $(22)$ can be computed efficiently. This algorithm can readily be adapted to obtain strategies to calculate the likelihoods defined by (32) and (35).

Algorithm 1: computation of likelihood $L(p)$ defined by (22):

- Step 1: initialization:

Choose a number $M C$ of Monte Carlo samples.

- Step 2: Monte Carlo simulation:

Simulate a set $\left\{\boldsymbol{\xi}_{s}, 1 \leq s \leq M C\right\}$ of $M C$ independent realizations of $\boldsymbol{\xi}$.

For each $s \in\{1 \leq s \leq M C\}$, use (21) to compute the realization

$$
\boldsymbol{a}_{s}^{\mathrm{r}}(\boldsymbol{p})=\sum_{\boldsymbol{\alpha},|\boldsymbol{\alpha}|=0}^{r} \boldsymbol{p}_{\boldsymbol{\alpha}} H_{\boldsymbol{\alpha}}\left(\boldsymbol{\xi}_{s}\right)
$$

\section{- Step 3: likelihood approximation:}

Estimate a PDF $f_{\boldsymbol{a}}^{\mathrm{MC}}\left(\cdot \mid \boldsymbol{p}^{\delta}\right)$ from the samples $\left\{\boldsymbol{a}_{s}^{\mathrm{r}}(\boldsymbol{p}), 1 \leq s \leq M C\right\}$.

Compute the likelihood as

$$
L^{\mathrm{MC}}(\boldsymbol{p})=\prod_{j=1}^{n} f_{\boldsymbol{a}}^{\mathrm{MC}}\left(\boldsymbol{a}^{(j)} \mid \boldsymbol{p}^{\delta}\right)
$$

Algorithm 1 requires in step 2 the computation of realizations of random variables. Methods for the simulation of random variables are surveyed in [32]. Step 3 requires the estimation of a PDF from a set of samples. The kernel density estimation method is used in this work [33-35]. We use the product kernel density estimation method for multivariate density estimation. And we use Scott's data-based rule-of-thumb 
for the estimation of the anisotropic kernel bandwidths, see e.g. Sec. 6.3 in [35].

Considering that the likelihood functions to be maximized in (23), (34) and (36) may have multiple local maxima and that it may be difficult to accurately calculate gradients with respect to the parameters, we suggest applying a global-search gradient-free optimization method. The simulated annealing $[32,36,37]$ and the genetic optimization method $[38,39]$ are natural choices. The latter is used here.

Algorithm 2 lists step by step how samples from the posterior PDF $\sigma(\boldsymbol{p})$ defined by (40) can be generated using the Gibbs Markov Chain Monte Carlo (MCMC) method, see e.g. [32]. This algorithm can readily be adapted to obtain a strategy to sample from posterior PDF $\sigma(\boldsymbol{p})$ defined by (37).

\section{Algorithm 2: MCMC sampling from $\sigma(p)$ defined by (40):}

\section{- Step 1: initialization:}

Choose a number $M C M C$ of MCMC samples and an initial value $\boldsymbol{p}^{(0)}=\left\{\boldsymbol{p}^{0(0)}, \boldsymbol{p}^{\delta(0)}\right\}$.

- Step 2: given $\left\{\boldsymbol{p}^{0(k)}, \boldsymbol{p}^{\delta(k)}\right\}$, generate $\left\{\boldsymbol{p}^{0(k+1)}, \boldsymbol{p}^{\delta(k)}\right\}$ :

For each $\ell \in\{1 \leq \ell \leq m\}$, sample $p_{\ell}^{0(k+1)}$, with reference to (39), from

$$
\sigma\left(p_{1}^{0(k+1)}, \ldots, p_{\ell-1}^{0(k+1)}, \cdot, p_{\ell+1}^{0(k)}, \ldots, p_{m}^{0(k)} \mid \boldsymbol{p}^{\delta(k)}\right)
$$

- Step 3: given $\left\{\boldsymbol{p}^{0(k+1)}, \boldsymbol{p}^{\delta(k)}\right\}$, generate $\left\{\boldsymbol{p}^{0(k+1)}, \boldsymbol{p}^{\delta(k+1)}\right\}$ :

For each $\ell \in\left\{1 \leq \ell \leq\left|\boldsymbol{p}^{\delta}\right|\right\}$, sample $p_{\ell}^{\delta(k+1)}$, with reference to (38), from

$$
\sigma\left(p_{1}^{\delta(k+1)}, \ldots, p_{\ell-1}^{\delta(k+1)}, \cdot, p_{\ell+1}^{\delta(k)}, \ldots, p_{\left|p^{\delta \mid}\right|}^{\delta(k)}\right)
$$

- Step 4: Repeat steps 2 and 3 for $k \in\{1 \leq k \leq M C M C\}$. 
Algorithm 2 requires in step 1 the choice of an initial value. We use the maximum likelihood estimate as starting point. Steps 2 and 3 involve the generation of samples from one-dimensional PDFs. Inverse transform sampling, see e.g. [32], is applied in this work. The required cumulative distribution function is hereby obtained by numerical integration of the PDF over a suitable subinterval of the real line.

Finally, it is noted that the coefficients $\boldsymbol{q}$ of PCE (43) can be estimated following a two-step procedure. First, samples from posterior PDF $\sigma(\boldsymbol{p})$ can be generated following algorithm 2. Then, the coefficients can in principle be estimated from these samples using the method of maximum likelihood.

\section{Illustration}

This section demonstrates the proposed methodology on a case history in stochastic aeroelastic stability analysis. The reader is referred to [40, 41] and references therein for more details concerning deterministic and stochastic aeroelastic stability analysis.

\subsection{Problem setting}

[Fig. 1 about here.]

Consider a collection of similar, but not perfectly identical, panels, each occupying at static equilibrium a box-shaped region

$$
\overline{\mathcal{D}}=\left\{-\frac{\ell}{2}<x_{1}<\frac{\ell}{2},-\frac{w}{2}<x_{2}<\frac{w}{2},-\frac{h}{2}<x_{3}<\frac{h}{2}\right\}
$$

in a Cartesian reference frame $\left(x_{1}, x_{2}, x_{3}\right)$ (Fig. 1). All panels are assumed to have identical in-plane dimensions $\ell$ and $w$, and thickness $h$. Let $\mathcal{D}$ be the middle plane 
such that $\overline{\mathcal{D}}=\mathcal{D} \times]-\frac{h}{2}, \frac{h}{2}[$. Let $\bar{\Gamma}=\partial \mathcal{D} \times]-\frac{h}{2}, \frac{h}{2}[$ denote the lateral boundary.

We are interested in the dynamical behavior of the panels while clamped along $\bar{\Gamma}$ and immersed in a supersonic flow in direction $x_{1}$. Since the panels are not perfectly identical, the flow velocity above which aerodynamic flutter instabilities occur is different for each panel. This illustration is concerned with the prediction of this scatter in the onset of flutter.

\subsection{Simulated data}

Data are synthetically generated using a stochastic model. The Kirchhoff-Love theory is used to represent the dynamical panel behavior, and the piston theory is applied to represent the forces exerted by the flow on the panel. Variability is accommodated by modeling fields of material properties by random fields.

The material is assumed elastic and isotropic. The Young's modulus is modeled by a lognormal random field $\left\{Y\left(x_{1}, x_{2}\right),\left(x_{1}, x_{2}\right) \in \mathcal{D}\right\}$ defined on $(\Omega, \mathcal{F}, P)$, indexed by $\mathcal{D}$ and with values in $\mathbb{R}_{0}^{+}$such that

$$
Y\left(x_{1}, x_{2}\right)=\underline{Y} \exp \left(\sqrt{\log \left(\delta^{2}+1\right)} g\left(x_{1}, x_{2}\right)-\frac{1}{2} \log \left(\delta^{2}+1\right)\right),
$$

in which $\underline{Y}$ is the mean, $\delta$ the coefficient of variation, and $\left\{g\left(x_{1}, x_{2}\right),\left(x_{1}, x_{2}\right) \in \mathcal{D}\right\}$ a Gaussian random field with zero mean and autocorrelation function

$$
\begin{aligned}
R_{g}(y 1, y 2) & =E\left\{g\left(x_{1}, x_{2}\right) g\left(x_{1}+y_{1}, x_{2}+y_{2}\right)\right\} \\
& =\frac{4 L^{2}}{\pi^{2} y_{1}^{2}} \sin ^{2}\left(\frac{\pi y_{1}}{2 L}\right) \frac{4 L^{2}}{\pi^{2} y_{2}^{2}} \sin ^{2}\left(\frac{\pi y_{2}}{2 L}\right),
\end{aligned}
$$

where $L$ is the spatial correlation length of the Gaussian random field. The Poisson ratio, denoted by $\nu$, is taken deterministic and homogeneous. Let the random field $\left\{D\left(x_{1} x_{2}\right),\left(x_{1}, x_{2}\right) \in \mathcal{D}\right\}$ represent the corresponding plate bending rigidity 
field such that

$$
D=\frac{Y h^{3}}{12\left(1-\nu^{2}\right)}
$$

Let $\rho$ denote the deterministic and homogeneous mass density.

Let $[0, T]$ be the time interval of interest. Let $u^{0}\left(x_{1}, x_{2}\right)$ and $u^{1}\left(x_{1}, x_{2}\right)$ be prescribed initial displacement and velocity fields. For a fixed realization, i.e. for a fixed $\omega \in \Omega$, the dynamical behavior of the panel is then described by an initial boundary value problem, which consists in finding the position- and time-dependent displacement field $u\left(x_{1}, x_{2} ; t, \omega\right)$ such that

$$
\left.\left(\frac{\partial^{2}}{\partial x_{1}^{2}}+\frac{\partial^{2}}{\partial x_{2}^{2}}\right)\left(D\left(\frac{\partial^{2} u}{\partial x_{1}^{2}}+\frac{\partial^{2} u}{\partial x_{2}^{2}}\right)\right)-q=-\rho h \frac{\partial^{2} u}{\partial t^{2}} \quad \text { in } \mathcal{D} \times\right] 0, T[
$$

with the boundary conditions

$$
\begin{array}{ll}
u=0 & \text { on } \partial \mathcal{D} \times] 0, T[ \\
\frac{\partial u}{\partial x_{1}}=0 & \text { on } \left.\partial \mathcal{D}\left(x_{1}= \pm \frac{\ell}{2}\right) \times\right] 0, T[ \\
\frac{\partial u}{\partial x_{2}}=0 & \text { on } \left.\partial \mathcal{D}\left(x_{2}= \pm \frac{w}{2}\right) \times\right] 0, T[
\end{array}
$$

and with the initial conditions

$$
\begin{array}{ll}
u\left(x_{1}, x_{2} ; 0\right)=u^{0}\left(x_{1}, x_{2}\right) & \text { in } \mathcal{D}, \\
\frac{\partial u}{\partial t}\left(x_{1}, x_{2} ; 0\right)=u^{1}\left(x_{1}, x_{2}\right) & \text { in } \mathcal{D} .
\end{array}
$$

Equation (53) is the classical Kirchhoff-Love plate equation. The piston theory models the force field excerted by the flow as

$$
q=-\frac{\rho_{\infty} v_{\infty}^{2}}{\sqrt{M_{\infty}^{2}-1}}\left(\frac{M_{\infty}^{2}-2}{M_{\infty}^{2}-1} \frac{1}{v_{\infty}} \frac{\partial u}{\partial t}+\frac{\partial u}{\partial x_{1}}\right)
$$

in which $\rho_{\infty}, v_{\infty}, a_{\infty}$ and $M_{\infty}=v_{\infty} / a_{\infty}$ are respectively the mass density, flow velocity, sound velocity and Mach number of the freestream flow. 
For a fixed realization, the weak formulation of (53)-(58) consists in finding the position- and time-dependent displacement field $u\left(x_{1}, x_{2} ; t ; \omega\right)$ such that for all sufficiently regular displacement fields $w\left(x_{1}, x_{2}\right)$ satisfying the boundary conditions (54)(56) and $\forall t \in] 0, T[$ :

$$
\int_{\mathcal{D}} \rho h \frac{\partial^{2} u}{\partial t^{2}} w d S+\int_{\mathcal{D}} D\left(\frac{\partial^{2} u}{\partial x_{1}^{2}} \frac{\partial^{2} w}{\partial x_{1}^{2}}+2 \frac{\partial^{2} u}{\partial x_{1} \partial x_{2}} \frac{\partial^{2} w}{\partial x_{1} \partial x_{2}}+\frac{\partial^{2} u}{\partial x_{2}^{2}} \frac{\partial^{2} w}{\partial x_{2}^{2}}\right) d S=\int_{\mathcal{D}} q w d S
$$

and the initial conditions (57)-(58) are fulfilled.

The Finite Element (FE) method is the natural choice for the spatial discretization of (60). Let the real and virtual displacement fields be expanded on FE basis functions $v_{j}$ as

$$
\begin{aligned}
u^{\mathrm{h}}\left(x_{1}, x_{2} ; t ; \omega\right) & =\sum_{j} u_{j}(t ; \omega) v_{j}\left(x_{1}, x_{2}\right), \\
w^{\mathrm{h}}\left(x_{1}, x_{2}\right) & =\sum_{j} w_{j} v_{j}\left(x_{1}, x_{2}\right) .
\end{aligned}
$$

For a fixed realization, the Galerkin projection of (60) reads as

$$
\boldsymbol{M} \frac{d^{2} \boldsymbol{u}}{d t^{2}}+\boldsymbol{K} \boldsymbol{u}+\boldsymbol{A}\left(v_{\infty}\right) \frac{d \boldsymbol{u}}{d t}+\boldsymbol{B}\left(v_{\infty}\right) \boldsymbol{u}=\mathbf{0}
$$

in which the mass matrix $\boldsymbol{M}$, stiffness matrix $\boldsymbol{K}$, aerodynamic damping matrix $\boldsymbol{A}\left(v_{\infty}\right)$ and aerodynamic stiffness matrix $\boldsymbol{B}\left(v_{\infty}\right)$ are defined by

$$
\begin{aligned}
& M_{j k}=\int_{\mathcal{D}} \rho h v_{k} v_{j} d S \\
& K_{j k}=\int_{\mathcal{D}} D\left(\frac{\partial^{2} v_{k}}{\partial x_{1}^{2}} \frac{\partial^{2} v_{j}}{\partial x_{1}^{2}}+2 \frac{\partial^{2} v_{k}}{\partial x_{1} x_{2}} \frac{\partial^{2} v_{j}}{\partial x_{1} x_{2}}+\frac{\partial^{2} v_{k}}{\partial x_{2}^{2}} \frac{\partial^{2} v_{j}}{\partial x_{2}^{2}}\right) d S \\
& A_{j k}\left(v_{\infty}\right)=\frac{\rho_{\infty} v_{\infty}^{2}}{\sqrt{M_{\infty}^{2}-1}} \frac{M_{\infty}^{2}-2}{M_{\infty}^{2}-1} \frac{1}{v_{\infty}} \int_{\mathcal{D}} v_{k} v_{j} d S \\
& B_{j k}\left(v_{\infty}\right)=\frac{\rho_{\infty} v_{\infty}^{2}}{\sqrt{M_{\infty}^{2}-1}} \int_{\mathcal{D}} \frac{\partial v_{k}}{\partial x_{1}} v_{j} d S
\end{aligned}
$$

The stability of system (63) can be explored using an eigenvalue approach. Equa- 
tion (63) is, for this purpose, rewritten in the following first-order form:

$$
\left[\begin{array}{cc}
\boldsymbol{M} & \mathbf{0} \\
\mathbf{0} & \boldsymbol{M}
\end{array}\right]\left[\begin{array}{c}
\frac{d^{2} \boldsymbol{u}}{d t^{2}} \\
\frac{d \boldsymbol{u}}{d t}
\end{array}\right]+\left[\begin{array}{cc}
\boldsymbol{A}\left(v_{\infty}\right) & \boldsymbol{K}+\boldsymbol{B}\left(v_{\infty}\right) \\
-\boldsymbol{M} & \mathbf{0}
\end{array}\right]\left[\begin{array}{l}
\frac{d \boldsymbol{u}}{d t} \\
\boldsymbol{u}
\end{array}\right]=\left[\begin{array}{l}
\mathbf{0} \\
\mathbf{0}
\end{array}\right]
$$

to which the following eigenvalue problem is associated:

$$
-\left[\begin{array}{cc}
\boldsymbol{A}\left(v_{\infty}\right) & \boldsymbol{K}+\boldsymbol{B}\left(v_{\infty}\right) \\
-\boldsymbol{M} & \mathbf{0}
\end{array}\right] \boldsymbol{\varphi}_{j}=\lambda_{j}\left[\begin{array}{cc}
\boldsymbol{M} & \mathbf{0} \\
\mathbf{0} & \boldsymbol{M}
\end{array}\right] \boldsymbol{\varphi}_{j} .
$$

The eigenvalues $\lambda_{j}$ can be shown to occur in complex conjugate pairs. If the real part of an eigenvalue pair is positive, then the coupled fluid-structure system is unstable.

Numerical results are presented for $\ell=1.25 \mathrm{~m}, w=1 \mathrm{~m}, h=0.003 \mathrm{~m}, \underline{Y}=70 \mathrm{GPa}$, $\delta=0.1, L=0.25 \mathrm{~m}, \nu=0.33, \rho=2700 \mathrm{~kg} / \mathrm{m}^{3}, \rho_{\infty}=0.45 \mathrm{~kg} / \mathrm{m}^{3}$, and $a_{\infty}=$ $295 \mathrm{~m} / \mathrm{s}$. The FE model is constituted of $25 \times 20$ plate elements of equal size.

[Fig. 2 about here.]

Figure 2 shows one realization of the random Young's modulus field.

[Fig. 3 about here.]

For this realization, Fig. 3 shows the real and imaginary part of the 20 lowest (by magnitude) eigenvalues of eigenproblem (69) as a function of the velocity of the freestream flow. It is observed that the real part of all eigenvalues is negative for velocities lower than about $580 \mathrm{~m} / \mathrm{s}$. However, beyond about $580 \mathrm{~m} / \mathrm{s}$, the real part of one pair of eigenvalues becomes positive. Hence, the system becomes unstable. The critical flow velocity is referred to as the flutter onset.

[Fig. 4 about here.] 
To illustrate this dynamic instability, Fig. 4 compares the response to an initial perturbation of the panel while submersed in a flow of velocity $500 \mathrm{~m} / \mathrm{s}$ to its response to the same initial perturbation while submersed in a flow of velocity $600 \mathrm{~m} / \mathrm{s}$. The below critical flow at $500 \mathrm{~m} / \mathrm{s}$ is observed to dampen the vibratory panel motion. In contrast, the above critical flow at $600 \mathrm{~m} / \mathrm{s}$ results in panel motion growing exponentially with time.

[Fig. 5 about here.]

A Monte Carlo analysis has been performed, which involved the calculation of the onset of flutter for a large number of realizations of the stochastic model. Figure 5 shows the PDF of the onset of flutter estimated from a sufficiently large number of those samples. This PDF is viewed as the PDF which ideally represents the scatter in the onset of flutter in the collection of panels under study.

[Table 1 about here.]

Three different data sets of the form (20) are generated using the stochastic model described above. They respectively collect $n=10, n=25$, and $n=500$ realizations of the eigenfrequencies of the 5 lowest-order longitudinal bending eigenmodes of the panel, solving the structural eigenproblem

$$
\boldsymbol{K} \boldsymbol{\varphi}_{j}=\lambda_{j}^{2} \boldsymbol{M} \boldsymbol{\varphi}_{j}
$$

With reference to $(20)$, each $\boldsymbol{a}^{(j)}$ is valued in $\left(\mathbb{R}^{+}\right)^{5}$ and collects 5 eigenfrequencies. Table 1 lists the data set of length $n=10$ (to allow the reader to reproduce results). 


\subsection{Stochastic model to be identified}

Let a deterministic FE model be built based upon the Kirchhoff-Love plate theory and the piston theory of the form

$$
\boldsymbol{M} \frac{d^{2} \boldsymbol{u}}{d t^{2}}+\underline{\boldsymbol{K}} \boldsymbol{u}+\boldsymbol{A}\left(v_{\infty}\right) \frac{d \boldsymbol{u}}{d t}+\boldsymbol{B}\left(v_{\infty}\right) \boldsymbol{u}=\mathbf{0}
$$

to describe the aerodynamical behavior of a panel with dimensions $\ell=1.25 \mathrm{~m}$, $w=1 \mathrm{~m}$ and $h=0.003 \mathrm{~m}$, Young's modulus $\underline{Y}=70 \mathrm{GPa}$, Poisson ratio $\nu=0.33$, and mass density $\rho=2700 \mathrm{~kg} / \mathrm{m}^{3}$, clamped along its lateral boundary, and submersed in a supersonic flow of velocity $v_{\infty}$, mass density $\rho_{\infty}=0.45 \mathrm{~kg} / \mathrm{m}^{3}$, and sound velocity $a_{\infty}=295 \mathrm{~m} / \mathrm{s}$. Let this model be projected onto the 5 lowest-order longitudinal bending eigenmodes of the structural eigenproblem

$$
\underline{\boldsymbol{K}} \boldsymbol{\varphi}_{j}=\lambda_{j}^{2} \boldsymbol{M} \boldsymbol{\varphi}_{j}
$$

to obtain a deterministic Reduced-Order Model (ROM) of the form

$$
\begin{aligned}
& \boldsymbol{M}^{\mathrm{red}} \frac{d^{2} \boldsymbol{q}}{d t^{2}}+\underline{\boldsymbol{K}}^{\mathrm{red}} \boldsymbol{q}+\boldsymbol{A}^{\mathrm{red}}\left(v_{\infty}\right) \frac{d \boldsymbol{q}}{d t}+\boldsymbol{B}^{\mathrm{red}}\left(v_{\infty}\right) \boldsymbol{q}=\mathbf{0}, \\
& \boldsymbol{u}=\boldsymbol{T} \boldsymbol{q}
\end{aligned}
$$

where the transformation matrix $\boldsymbol{T}$ defines the reduction basis, matrices $\boldsymbol{M}^{\mathrm{red}}=$ $\boldsymbol{T}^{\mathrm{T}} \boldsymbol{M} \boldsymbol{T}, \underline{\boldsymbol{K}}^{\mathrm{red}}=\boldsymbol{T}^{\mathrm{T}} \underline{\boldsymbol{K}} \boldsymbol{T}, \boldsymbol{A}^{\mathrm{red}}\left(v_{\infty}\right)=\boldsymbol{T}^{\mathrm{T}} \boldsymbol{A}\left(v_{\infty}\right) \boldsymbol{T}^{\mathrm{T}}$ and $\boldsymbol{B}^{\mathrm{red}}\left(v_{\infty}\right)=\boldsymbol{T}^{\mathrm{T}} \boldsymbol{B}\left(v_{\infty}\right) \boldsymbol{T}$ are the reduced mass, stiffness, aerodynamic damping and aerodynamic stiffness matrices, and, for a fixed $t, \boldsymbol{q}(t)$ is the vector of the generalized coordinates.

Let uncertainty be accommodated by modeling the structural eigenfrequencies by random variables $a_{j}$, while keeping the structural eigenmodes deterministic, to ob- 
tain a stochastic ROM of the form:

$$
\begin{aligned}
& \boldsymbol{M}^{\mathrm{red}} \frac{d^{2} \boldsymbol{q}}{d t^{2}}+\operatorname{Diag}\left(\left(2 \pi a_{j}\right)^{2}\right) \boldsymbol{q}+\boldsymbol{A}^{\mathrm{red}}\left(v_{\infty}\right) \frac{d \boldsymbol{q}}{d t}+\boldsymbol{B}^{\mathrm{red}}\left(v_{\infty}\right) \boldsymbol{q}=\mathbf{0}, \\
& \boldsymbol{u}=\boldsymbol{T} \boldsymbol{q}
\end{aligned}
$$

in which $\operatorname{Diag}\left(\left(2 \pi a_{j}\right)^{2}\right)$ is a diagonal random matrix with diagonal elements $\left(2 \pi a_{j}\right)^{2}$, and the matrices $\boldsymbol{T}, \boldsymbol{M}^{\text {red }}, \boldsymbol{A}^{\text {red }}\left(v_{\infty}\right)$ and $\boldsymbol{B}^{\text {red }}\left(v_{\infty}\right)$ are deterministic.

Let the random variables $a_{j}$ be collected in a random vector $\boldsymbol{a}$ with values in $\left(\mathbb{R}^{+}\right)^{5}$. A reduced representation of $\boldsymbol{a}$ will be identified from each data set next.

\subsection{Maximum likelihood stochastic inversion}

The methology presented in Sec. 6.3 is followed to approximate, for each data set, random variable $\boldsymbol{a}$ by a reduced representation of the form

$$
\boldsymbol{a}^{\mathrm{d}, \mathrm{r}}(\boldsymbol{p})=\exp \left(\boldsymbol{p}^{0}+\sum_{j=1}^{d} \sqrt{\hat{\lambda}_{j}} \sum_{\boldsymbol{\alpha},|\boldsymbol{\alpha}|=1}^{r} p_{\boldsymbol{\alpha} j}^{\delta} H_{\boldsymbol{\alpha}}(\boldsymbol{\xi}) \hat{\boldsymbol{v}}_{j}\right),
$$

in which $\hat{\lambda}_{j}$ and $\hat{\boldsymbol{v}}_{j}$ denote the eigenvalues and eigenvectors of the sample covariance matrix, random variable $\boldsymbol{\xi}$ with values in $\mathbb{R}^{d}$ is chosen to have independent standard Gaussian components, and $H_{\boldsymbol{\alpha}}$ is the multidimensional Hermite polynomial of multiindex $\boldsymbol{\alpha}$. The exponential serves to ensure positivity.

[Fig. 6 about here.]

Figure 6 shows the eigenvalues of the sample covariance matrices, suggesting that, for each data set, a reduced representation of dimension $d=2$ is able to represent more than $95 \%$ of the variability in the eigenfrequencies. All results to follow have been obtained with dimension $d=2$ and order $r=1$.

[Fig. 7 about here.] 
[Table 2 about here.]

The methodology presented in Sec. 6.4 is followed to infer, from each data set, optimal coefficients $\hat{\boldsymbol{p}}^{0}$ and $\hat{\boldsymbol{p}}^{\delta}$ using the method of maximum likelihood. This involves the numerical approximation of the PDFs $f_{\boldsymbol{\eta}}\left(\cdot \mid \boldsymbol{p}^{\delta}\right)$ and $f_{a_{k}}\left(\cdot \mid \boldsymbol{p}^{0}, \hat{\boldsymbol{p}}^{\delta}\right)$ by the kernel density estimation method from samples generated by Monte Carlo simulation. Figure 7 shows the $\log$ likelihood $\log L^{\delta}\left(\boldsymbol{p}^{\delta}\right)$ defined by (32) as a function of the number $M C$ of Monte Carlo samples for the data set of length 25 and a specified value of $\boldsymbol{p}^{\delta}$. Reasonable convergence is obtained for $M C=10000$. All results to follow have been obtained using $M C=10000$ samples. Table 2 lists the optimal coefficients identified from the data set of length 10 .

[Fig. 8 about here.]

Each of the three identified sets of optimal coefficients defines via (75)-(76) and (77) a stochastic ROM. Each of these ROMs has been used to predict a PDF for the onset of flutter by Monte Carlo simulation. Figure 8 compares the three PDFs thus obtained to the ideal PDF predicted by the data-generating model.

As the length of the data set increases to infinity, the PDF predicted by the identified stochastic ROM can be expected to converge to some asymptotic PDF. However, the latter should be expected to differ from the ideal PDF predicted by the datagenerating model due to modeling errors associated with the dimension reductions performed while constructing the stochastic ROM. 


\subsection{Bayesian stochastic inversion}

The methodology presented in Sec. 7.2 will now be followed to construct posterior PDFs over $\boldsymbol{p}^{0}$ and $\boldsymbol{p}^{\delta}$. The improper uniform PDF over the coefficients is hereby used as a noninformative prior.

[Fig. 9 about here.]

For each data set, algorithm 2 is implemented to sample from the posterior by MCMC simulation. Low-order marginal posterior PDFs can efficiently be estimated from these samples by kernel density estimation. Figure 9 shows the first-order marginal posterior PDF thus obtained over $p_{1}^{0}$ for the data set of length 25 as a function of the number $M C M C$ of samples. Reasonable convergence is obtained for $M C M C=5000$. All results to follow have been obtained using respectively 10000 , 5000 and 250 samples for the inferences from the data sets of lengths 10, 25 and 500.

[Fig. 10 about here.]

Figure 10 shows for each data set the first-order marginal posterior PDF over $p_{1}^{0}$. This PDFs is interpreted as a quantitative description of the undertainty in $p_{1}^{0}$ due to the finite length of the data set. It is observed that better knowledge of $p_{1}^{0}$ is acquired as more data is collected.

[Table 3 about here.]

[Fig. 11 about here.]

Table 3 lists 5 realizations of $\boldsymbol{p}^{0}$ and $\boldsymbol{p}^{\delta}$ sampled from the posterior associated with the data set of length 10. Each of these realizations corresponds via (75)(76) and (77) to a stochastic ROM, which has been used to predict a PDF for the 
onset of flutter by Monte Carlo simulation. Figure 11(a) compares the PDFs thus obtained to the ideal PDF predicted by the data-generating model. Figures 11(b) and 11(c) are similar figures for the data sets of lengths 25 and 500 .

[Fig. 12 about here.]

Figure 12 shows, for each data set, a Bayesian posterior 99\%-confidence region for the PDF for the onset of flutter. It is such that the realizations of the latter PDF corresponding to realizations of $\boldsymbol{p}^{0}$ and $\boldsymbol{p}^{\delta}$ sampled from the posterior PDF (cfr. Fig. 11) lie within this region with a probability exceeding 99\%. This confidence region is interpreted as a quantitative description of the undertainty in the predicted PDF for the onset of flutter due to the finite length of the data set. Better knowledge of this PDF is acquired as more data is gathered.

\section{Conclusion}

We have developed a Bayesian inverse methodology for the identification of PCEs from experimental data. The procedure provides a quantitative characterization of the impact of missing experimental information on the accuracy of the identified PCEs, and can hence be used to estimate the value of additional data, as required for resource allocation aimed at improving predictions.

\section{Acknowledgements}

This work was supported by NSF, Sandia National Laboratories, and a MURI administered by AFOSR on Health Monitoring and Materials Damage Prognosis for Metallic Aerospace Propulsion and Structural Systems. 


\section{References}

[1] R.A. Ibrahim. Structural dynamics with parameter uncertainties. ASME Applied Mechanics Reviews, 40:309-328, 1987.

[2] C.S. Manohar and R.A. Ibrahim. Progress in structural dynamics with stochastic parameter variations 1987-1998. ASME Applied Mechanics Reviews, 52: 177-197, 1999.

[3] G.I. Schueller, L.A. Bergman, C.G. Bucher, G. Dasgupta, G. Deotdatis, R.G. Ghanem, M. Grigoriu, M. Hoshiya, E.A. Johnson, N.A. Naess, H.J. Pradlwarter, M. Shinozuka, K. Sobszyck, P.D. Spanos, B.F. Spencer, A. Sutoh, T. Takada, W.V. Wedig, S.F. Wojtkiewicz, I. Yoshida, B.A. Zeldin, and R. Zhang. A state-of-the-art report on computational stochastic mechanics. Probabilistic Engineering Mechanics, 12:197-321, 1997.

[4] G.I. Schueller. Computational stochastic mechanics - recent advances. Computers and Structures, 79:2225-2234, 2001.

[5] R. Ghanem and P. Spanos. Stochastic Finite Elements:A Spectral Approach. Springer, 1991.

[6] C. Soize. A non-parametric model of random uncertainties for reduced matrix models in structural dynamics. Probabilistic Engineering Mechanics, 15:277$294,2000$.

[7] C. Soize. Maximum entropy approach for modeling random uncertainties in transient elastodynamics. Journal of the Acoustical Society of America, 109: 1979-1996, 2001.

[8] C. Soize and R. Ghanem. Physical systems with random uncertainties: chaos representations with arbitrary probability measure. SIAM Journal on Scientific Computing, 26:395-410, 2004.

[9] C. Soize and R. Ghanem. Reduced chaos decomposition with random coeffi- 
cients of vector-valued random variables and random fields. Computer Methods in Applied Mechanics and Engineering, 2009. In Press.

[10] N. Wiener. The homogeneous chaos. Americal Journal of Mathematics, 60: 897-936, 1938.

[11] D. Xiu and G.E. Karniadakis. The Wiener-Askey polynomial chaos for stochastic differential equations. SIAM Journal on Scientific Computing, 24:619-644, 2002.

[12] S. Kullback. Information Theory and Statistics. Dover Publications, 1968.

[13] H. Cramér. Mathematical Methods of Statistics. Princeton University Press, 1946.

[14] M. Arnst and R. Ghanem. Probabilistic equivalence and stochastic model reduction in multiscale analysis. Computer Methods in Applied Mechanics and Engineering, 197:3584-3592, 2008.

[15] C. Soize, E. Capiez Lernout, J. Durand, C. Fernandez, and L. Gagliardini. Probabilistic model identification of uncertainties in computational models for dynamical systems and experimental validation. Computer Methods in Applied Mechanics and Engineering, 1:150-163, 2008.

[16] M. Arnst, D. Clouteau, and M. Bonnet. Inversion of probabilistic structural models using measured transfer functions. Computer Methods in Applied Mechanics and Engineering, 197:589-608, 2008.

[17] C. Desceliers, R. Ghanem, and C. Soize. Maximum likelihood estimation of stochastic chaos representations from experimental data. International Journal for Numerical Methods in Engineering, 66:978-1001, 2006.

[18] R. Ghanem, A. Doostan, and J. Red Horse. A probabilistic construction of model validation. Computer Methods in Applied Mechanics and Engineering, 197:2585-2595, 2008.

[19] M. Hübner and B.L. Rozovskii. On asymptotic properties of maximum likeli- 
hood estimators for parabolic stochastic PDEs. Probability and Related Fields, 103:143-163, 1995.

[20] Y. Marzouk, H. Najm, and L. Rahn. Stochastic spectral methods for efficient Bayesian solution of inverse problems. Journal of Computational Physics, 224: 560-586, 2007.

[21] Y. Marzouk and D. Xiu. A stochastic collocation approach to Bayesian inference in inverse problems. Communications in Computational Physics, 6:826-847, 2009.

[22] Y. Marzouk and H. Najm. Dimensionality reduction and polynomial chaos acceleration of Bayesian inference in inverse problems. Journal of Computational Physics, 228:1862-1902, 2009.

[23] S. Das, R. Ghanem, and J. Spall. Asymptotic sampling distribution for polynomial chaos representation of data: A maximum entropy and fisher information approach. SIAM Journal on Scientific Computing, 28:2207-2234, 2008.

[24] R. Ghanem and A. Doostan. On the construction and analysis of stochastic models: Characterization and propagation of the errors associated with limited data. Journal of Computational Physics, 217:63-81, 2006.

[25] R.H. Cameron and W.T. Martin. The orthogonal development of nonlinear functionals in series of Fourier-Hermite functionals. Annals of Mathematics, 48:385-392, 1947.

[26] A. Tarantola. Inverse Problem Theory and Methods for Model Parameter Estimation. SIAM, 2005.

[27] A. Gelman, J. Carlin, H. Stern, and D. Rubin. Bayesian Data Analysis. Chapman \& Hall/CRC, 2003.

[28] J. Bernardo and A. Smith. Bayesian theory. Wiley, 2000.

[29] E. Jaynes. Probability Theory: The Logic of Science. Cambridge University Press, 2003. 
[30] C. Soize. Construction of probability distributions in high dimension using the maximum entropy principle: applications to stochastic processes, random fields and random matrices. International Journal for Numerical Methods in Engineering, 76:15831611, 2008.

[31] J.R. Red-Horse and A.S. Benjamin. A probabilistic approach to uncertainty quantification with limited information. Reliability Engineering $\&$ System Safety, 85:183-190, 2004.

[32] C.P. Robert and G. Casella. Monte Carlo Statistical Methods. Springer, 2005.

[33] E. Parzen. On estimation of probability density function and mode. Annals of Mathematical Statistics, 33:1065-1076, 1962.

[34] M. Rosenblatt. Remarks on some nonparametric estimates of a density function. Annals of Mathematical Statistics, 27:832-837, 1956.

[35] D.W. Scott. Multivariate Density Estimation: Theory, Practice, and Visualization. Wiley-Interscience, 1992.

[36] S. Kirkpatrick, C.D. Gelatt, and M.P. Vecchi. Optimization by simulated annealing. Science, 220:671-680, 1983.

[37] N. Metropolis, A.W. Rosenbluth, M.N. Rosenbluth, A.H. Teller, and E. Teller. Equations of state calculations by fast computing machines. The Journal of Chemical Physics, 21:1087-1092, 1953.

[38] D.E. Goldberg. Genetic Algorithms in Search, Optimization and Machine Learning. Addison-Wesley Professional, 1989.

[39] D.B. Fogel. Evolutionary Computation : Towards a New Philosophy of Machine Intelligence. IEEE Computer Society Press, 1995.

[40] E.H. Dowell. Aeroelasticity of plates and shells. Noordhoff International, 1974.

[41] N.J. Lindsley, C.L. Pettit, and P.S. Beran. Nonlinear plate aeroelastic response with uncertain stiffness and boundary conditions. Structure and Infrastructure Engineering, 2:201-220, 2006. 


\section{List of Figures}

1 Problem setting: schematic representation of the panel in the supersonic flow.

2 Simulated data: one realization of the random Young's modulus field.

3 Simulated data: real and imaginary part of the 20 lowest (by magnitude) eigenvalues of eigenproblem (69) as a function of the freestream flow velocity $v_{\infty}$.

4 Simulated data: transient vertical response at the center of the panel due to an initial perturbation for freestream flow velocity (a) $500 \mathrm{~m} / \mathrm{s}$ and (b) $600 \mathrm{~m} / \mathrm{s}$.

$5 \quad$ Simulated data: ideal PDF for the onset of flutter.

6 Maximum likelihood stochastic inversion: eigenvalues of the sample covariance matrices associated with the data sets of length 10 (dotted line), 25 (dashed line) and 500 (solid line).

7 Maximum likelihood stochastic inversion: $\log$ likelihood $\log L^{\delta}\left(\boldsymbol{p}^{\delta}\right)$ as a function of the number $M C$ of Monte Carlo samples for the data set of length 25 and $p_{101}^{\delta}=-0.9837, p_{102}^{\delta}=-0.0209, p_{011}^{\delta}=-0.0035$ and $p_{012}^{\delta}=-0.8734$.

8 Maximum likelihood stochastic inversion: PDF for the onset of flutter predicted by the stochastic ROM identified from the data set of length (a) 10, (b) 25 and (c) 500 (dashed line), and ideal PDF predicted by the data-generating model (solid line).

9 Bayesian stochastic inversion: first-order marginal posterior PDF over $p_{1}^{0}$ for the data set of length 25 estimated from 10 (dotted line), 100 (dash-dotted line), 1000 (dashed line), and 5000 (solid line) MCMC samples.

10 Bayesian stochastic inversion: first-order marginal posterior PDF over $p_{1}^{0}$ associated with the data set of length (a) 10, (b) 25 and (c) 500 .

11 Bayesian stochastic inversion: 5 PDFs for the onset of flutter predicted by 5 stochastic ROMs corresponding to 5 sets of coefficients sampled from the posterior PDF associated with the data set of length (a) 10, (b) 25 and (c) 500 (dashed lines), and ideal PDF predicted by the data-generating model (solid line). 
12 Bayesian stochastic inversion: posterior 99\%-confidence region for the PDF for the onset of flutter for the data set of length (a) 10, (b) 25 and (c) 500 (filled), and ideal PDF predicted by the data-generating model (solid line). 


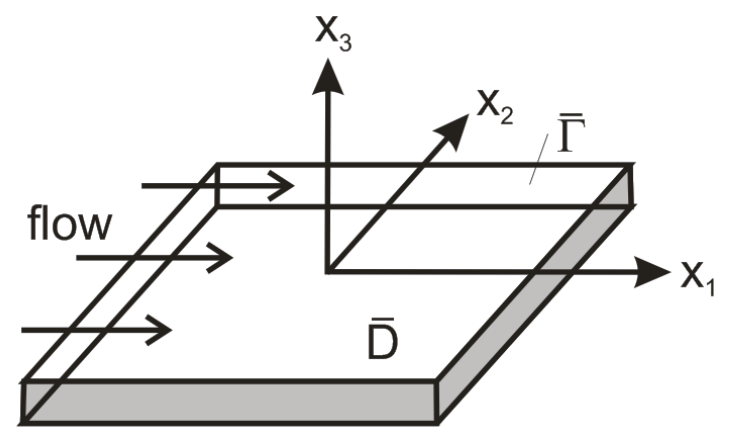

Fig. 1. Problem setting: schematic representation of the panel in the supersonic flow. 


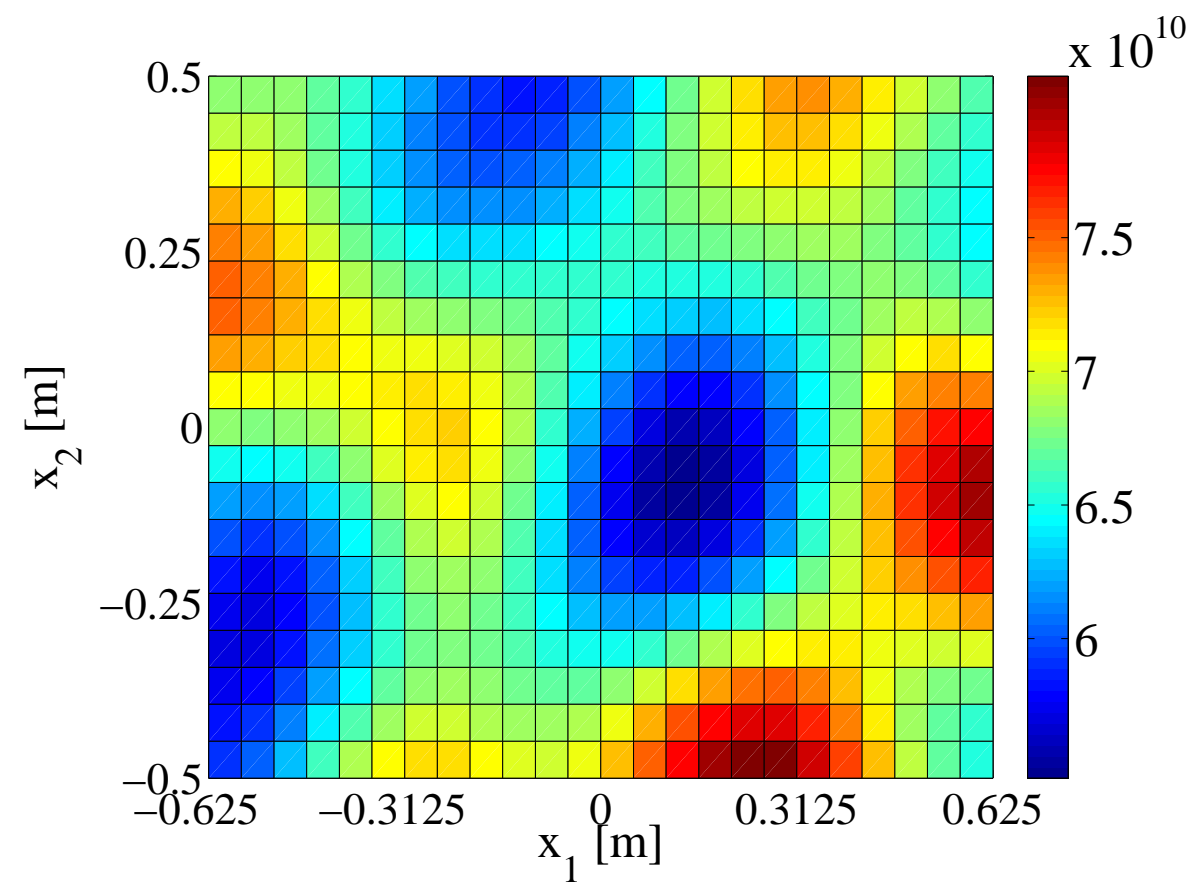

Fig. 2. Simulated data: one realization of the random Young's modulus field. 


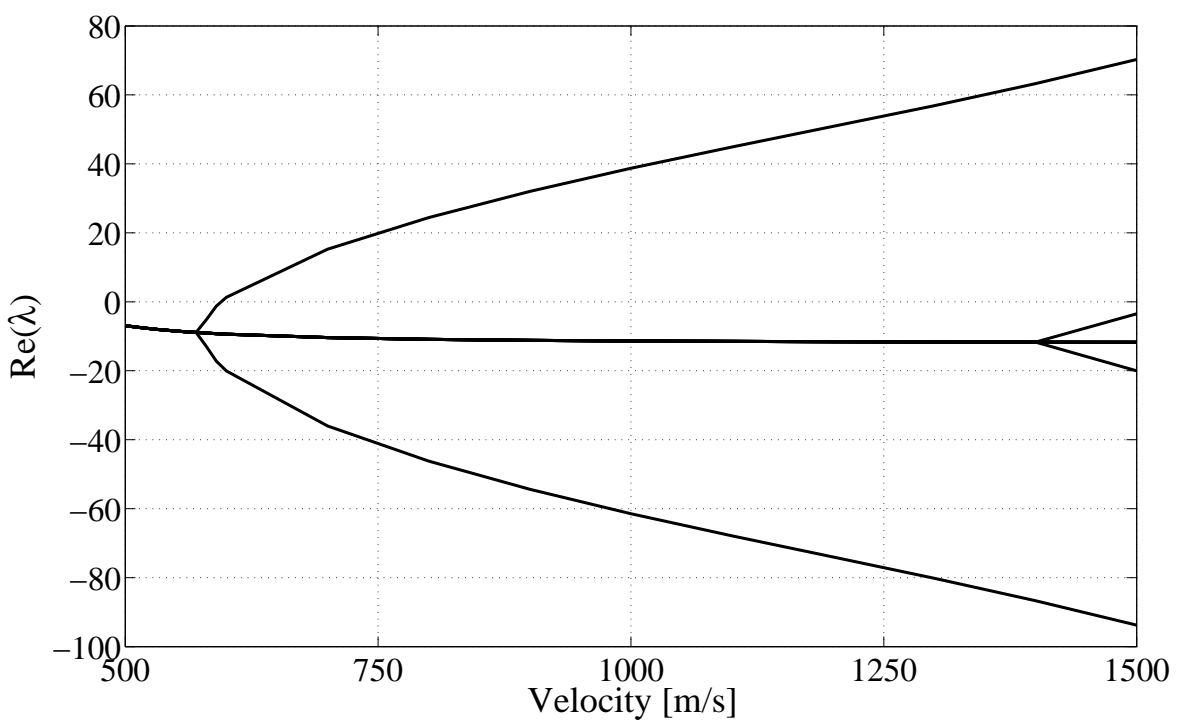

(a) Real part.

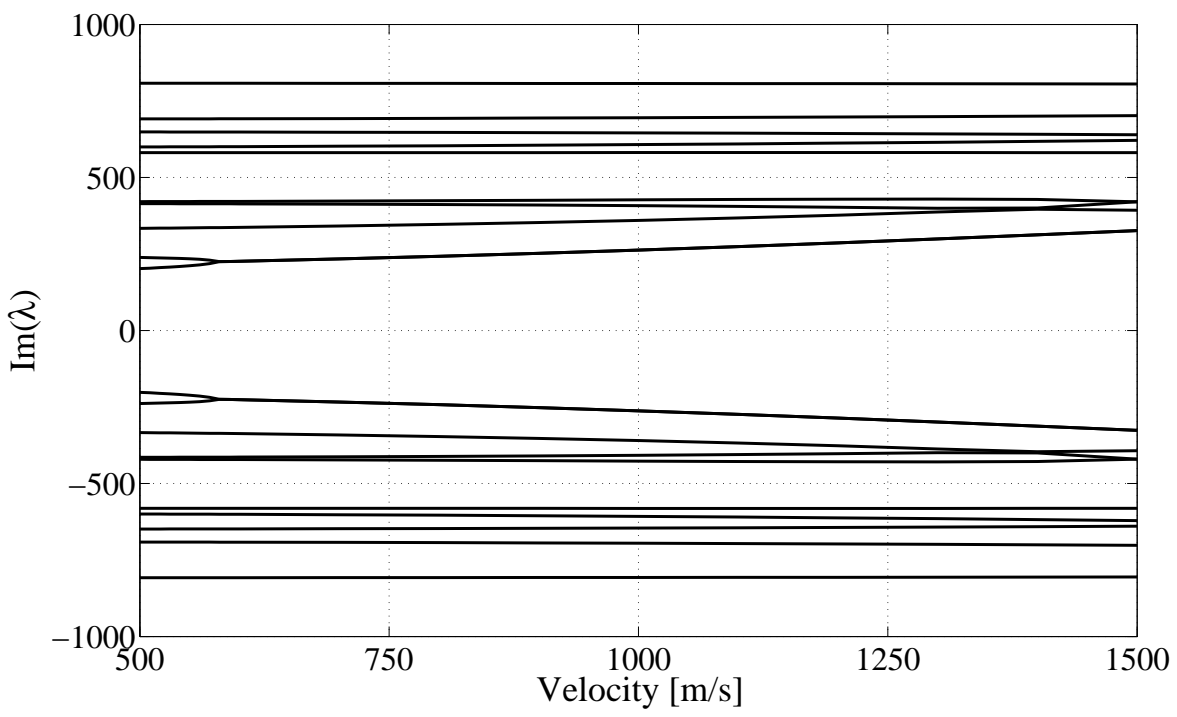

(b) Imaginary part.

Fig. 3. Simulated data: real and imaginary part of the 20 lowest (by magnitude) eigenvalues of eigenproblem (69) as a function of the freestream flow velocity $v_{\infty}$. 


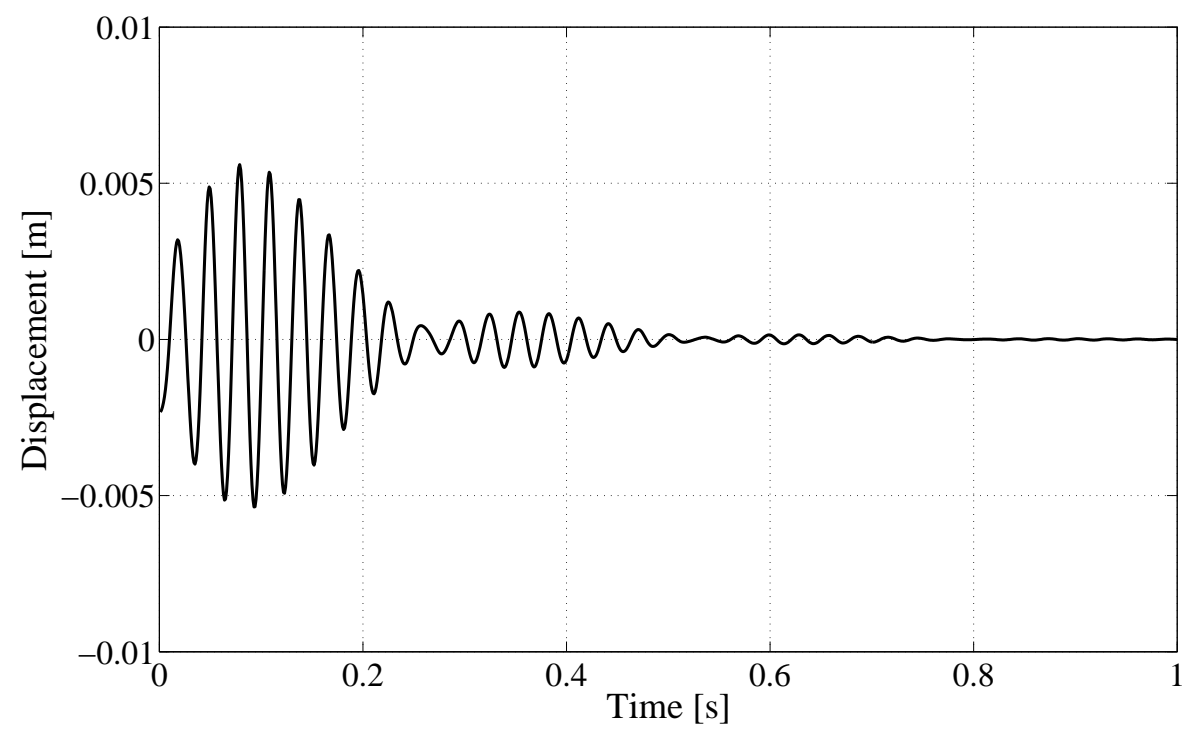

(a) $v_{\infty}=500 \mathrm{~m} / \mathrm{s}$.

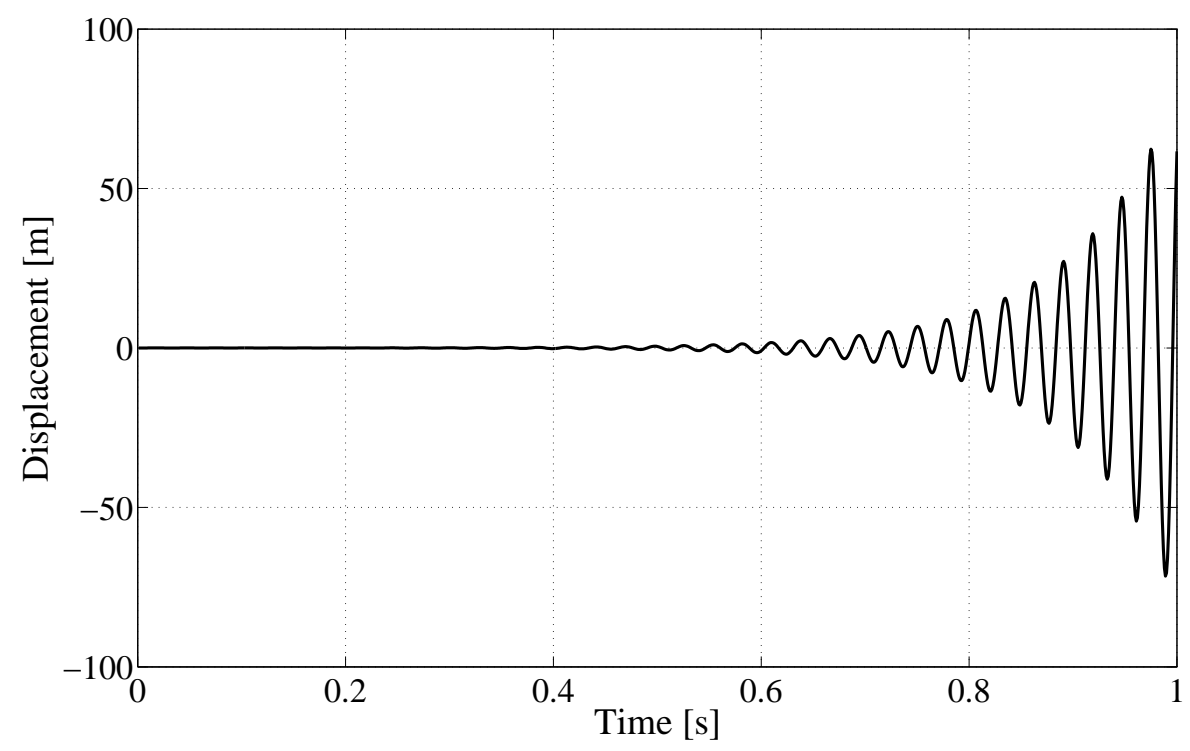

(b) $v_{\infty}=600 \mathrm{~m} / \mathrm{s}$.

Fig. 4. Simulated data: transient vertical response at the center of the panel due to an initial perturbation for freestream flow velocity (a) $500 \mathrm{~m} / \mathrm{s}$ and (b) $600 \mathrm{~m} / \mathrm{s}$. 


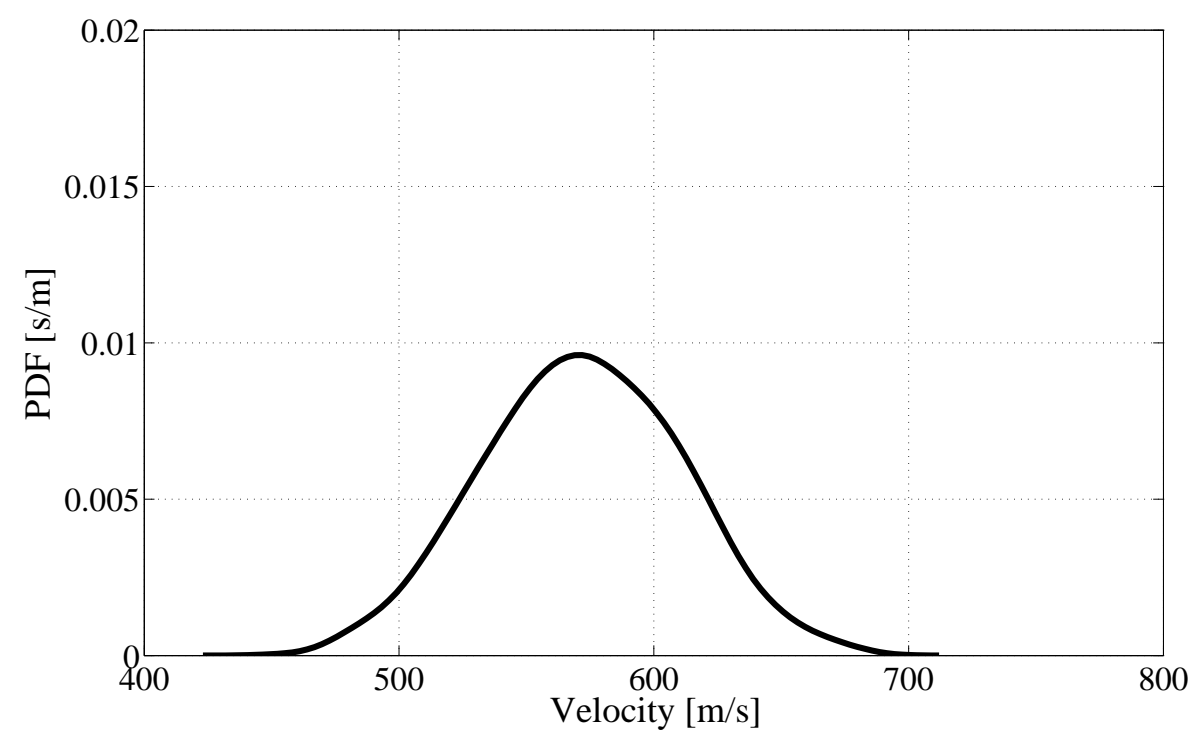

(a)

Fig. 5. Simulated data: ideal PDF for the onset of flutter. 


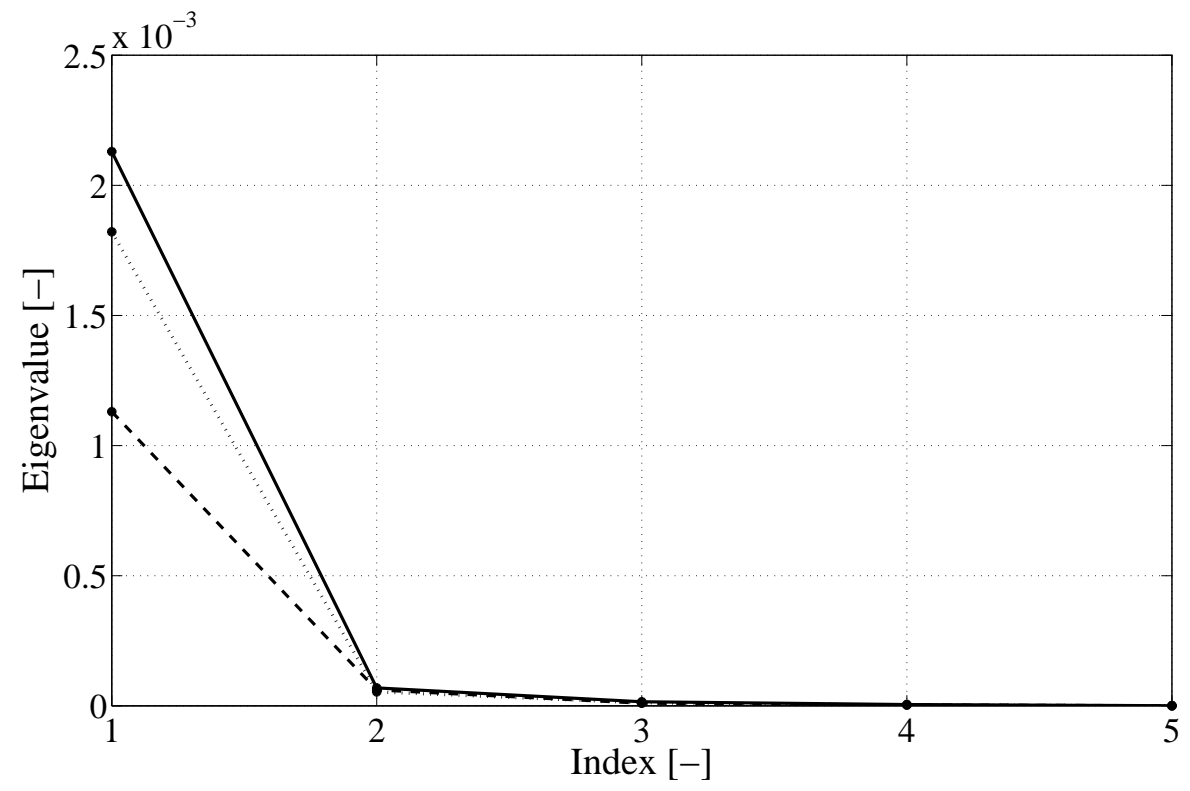

Fig. 6. Maximum likelihood stochastic inversion: eigenvalues of the sample covariance matrices associated with the data sets of length 10 (dotted line), 25 (dashed line) and 500 (solid line). 


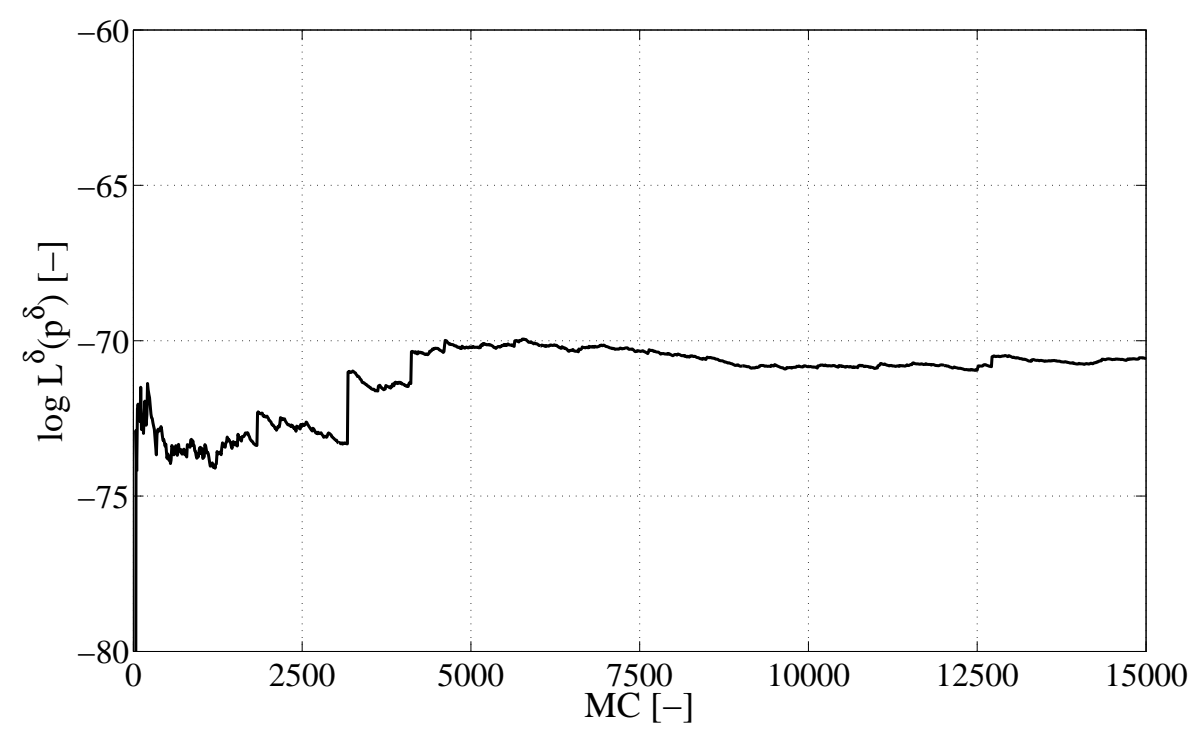

Fig. 7. Maximum likelihood stochastic inversion: $\log$ likelihood $\log L^{\delta}\left(\boldsymbol{p}^{\delta}\right)$ as a function of the number $M C$ of Monte Carlo samples for the data set of length 25 and $p_{101}^{\delta}=-0.9837$, $p_{102}^{\delta}=-0.0209, p_{011}^{\delta}=-0.0035$ and $p_{012}^{\delta}=-0.8734$. 


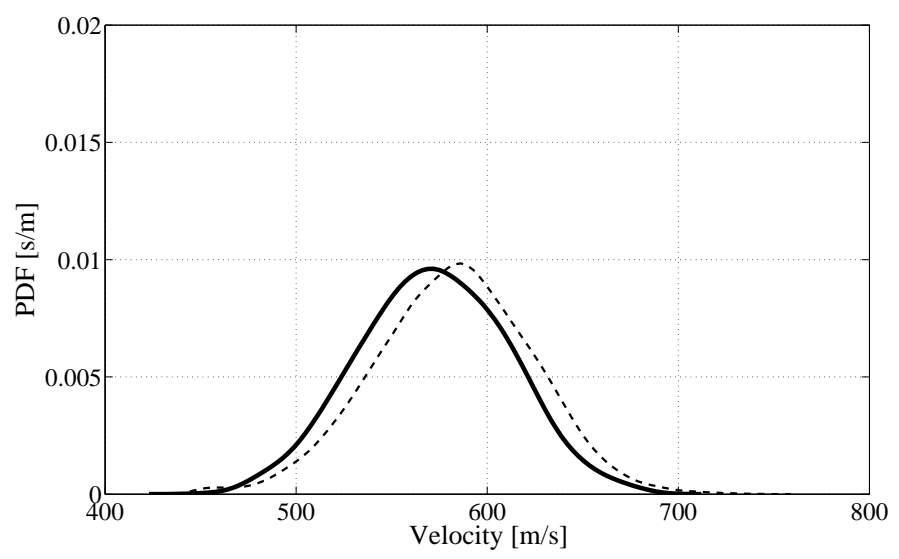

(a) $n=10$.

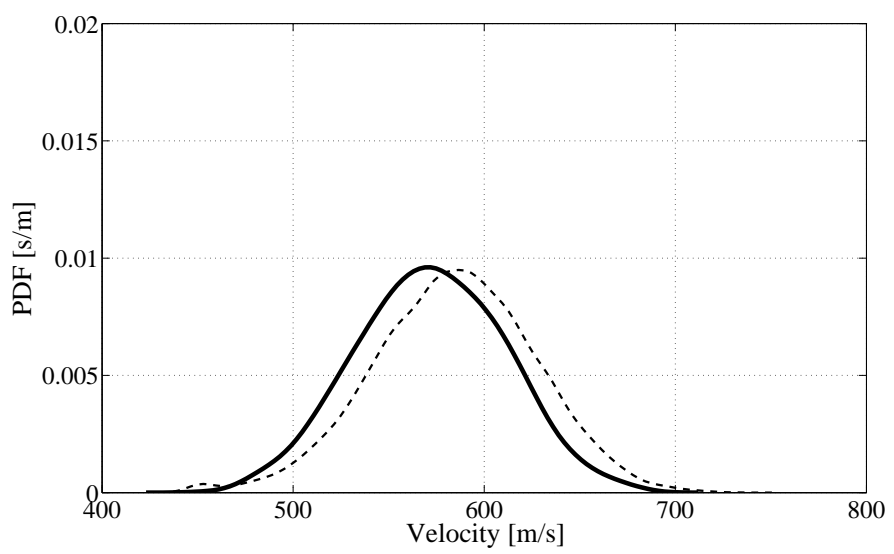

(b) $n=25$.

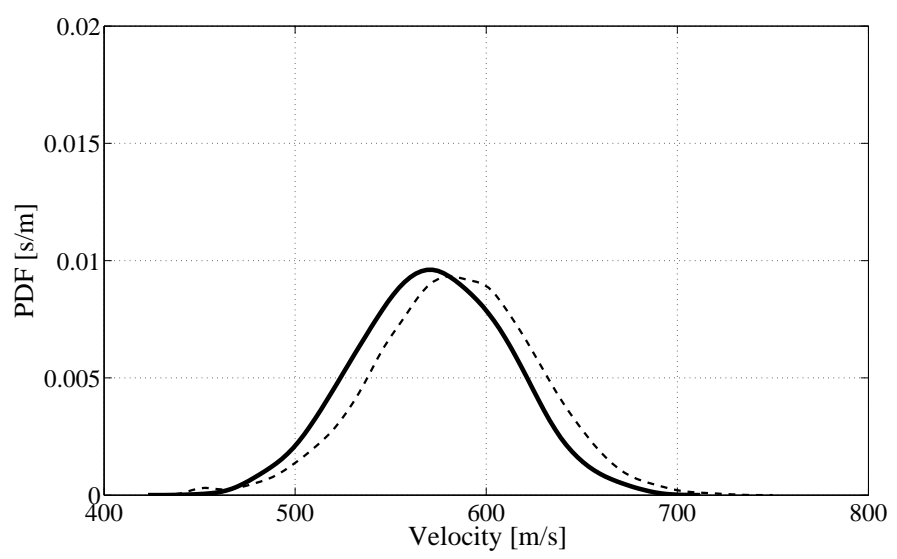

(c) $n=500$.

Fig. 8. Maximum likelihood stochastic inversion: PDF for the onset of flutter predicted by the stochastic ROM identified from the data set of length (a) 10, (b) 25 and (c) 500 (dashed line), and ideal PDF predicted by the data-generating model (solid line). 


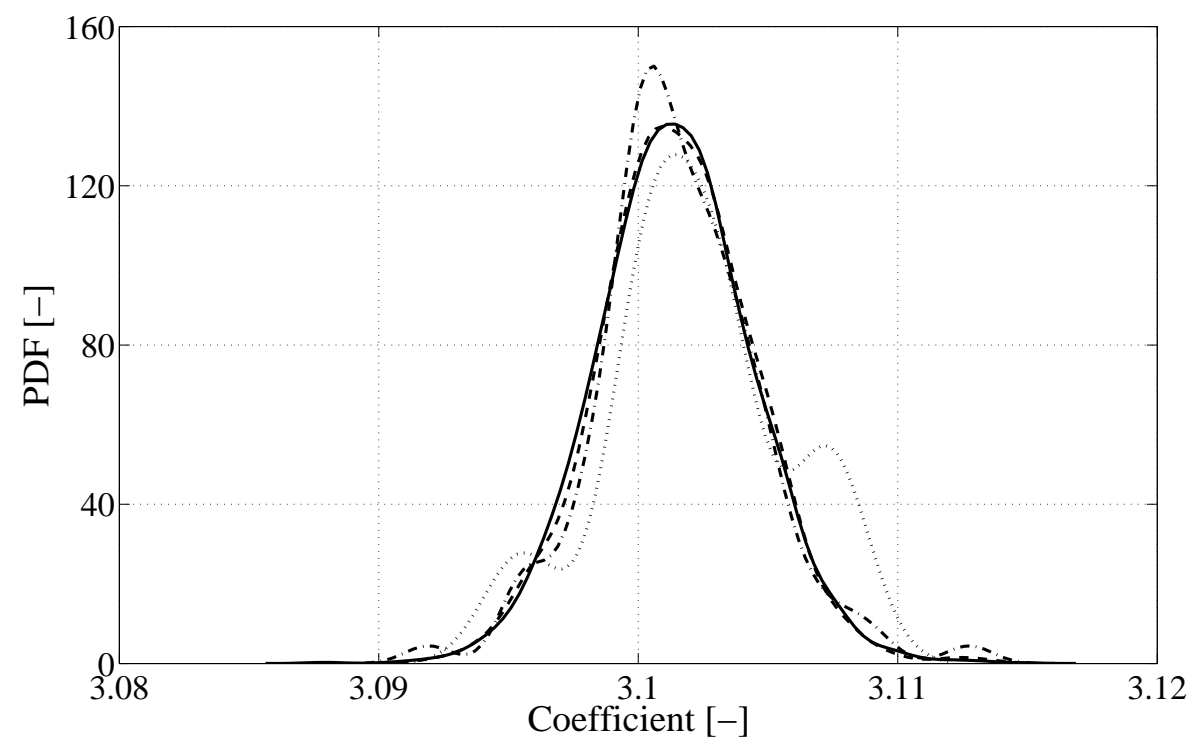

Fig. 9. Bayesian stochastic inversion: first-order marginal posterior PDF over $p_{1}^{0}$ for the data set of length 25 estimated from 10 (dotted line), 100 (dash-dotted line), 1000 (dashed line), and 5000 (solid line) MCMC samples. 


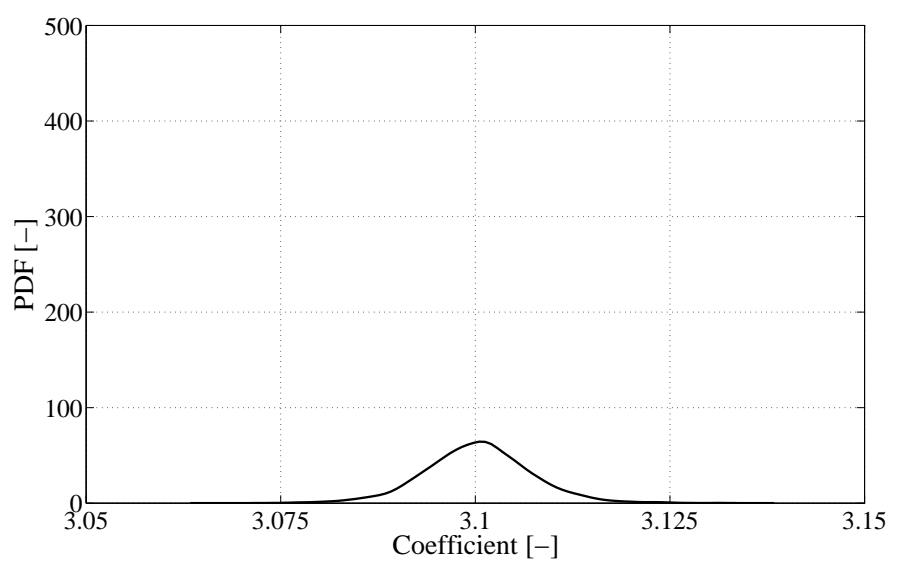

(a) $n=10$.

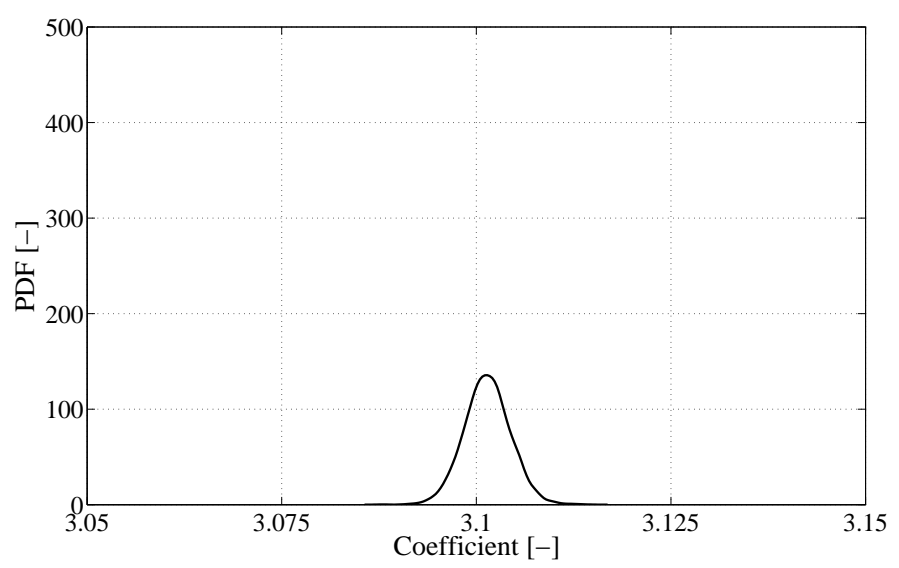

(b) $n=25$.

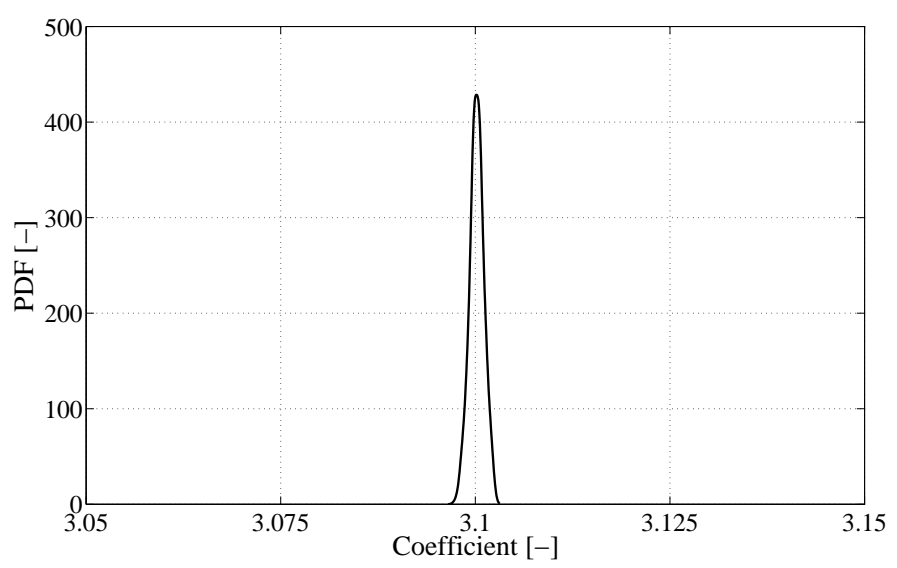

(c) $n=500$.

Fig. 10. Bayesian stochastic inversion: first-order marginal posterior PDF over $p_{1}^{0}$ associated with the data set of length (a) 10, (b) 25 and (c) 500. 


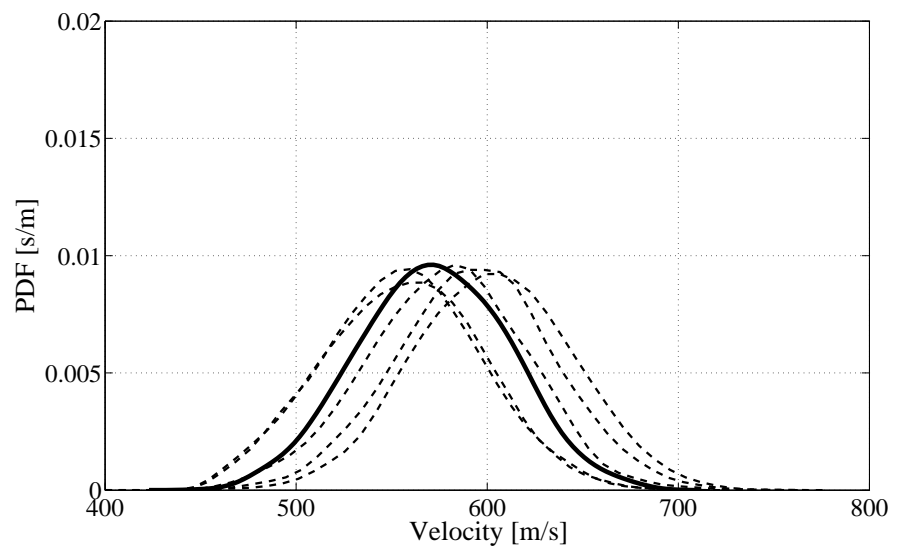

(a) $n=10$.

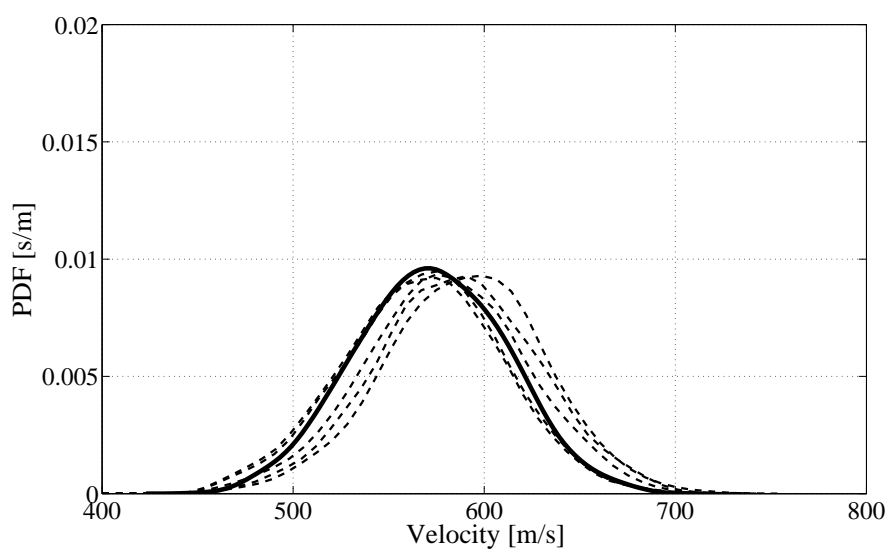

(b) $n=25$.

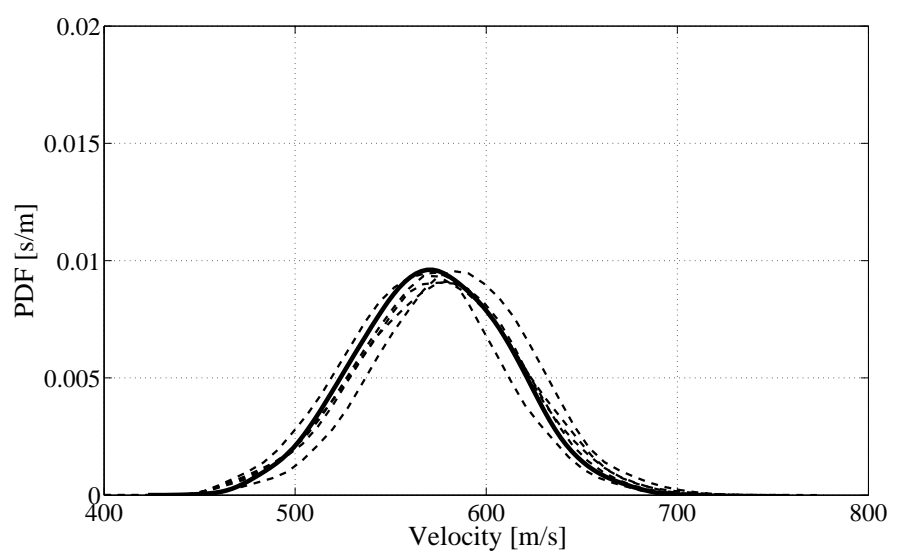

(c) $n=500$.

Fig. 11. Bayesian stochastic inversion: 5 PDFs for the onset of flutter predicted by 5 stochastic ROMs corresponding to 5 sets of coefficients sampled from the posterior PDF associated with the data set of length (a) 10, (b) 25 and (c) 500 (dashed lines), and ideal PDF predicted by the data-generating model (solid line). 


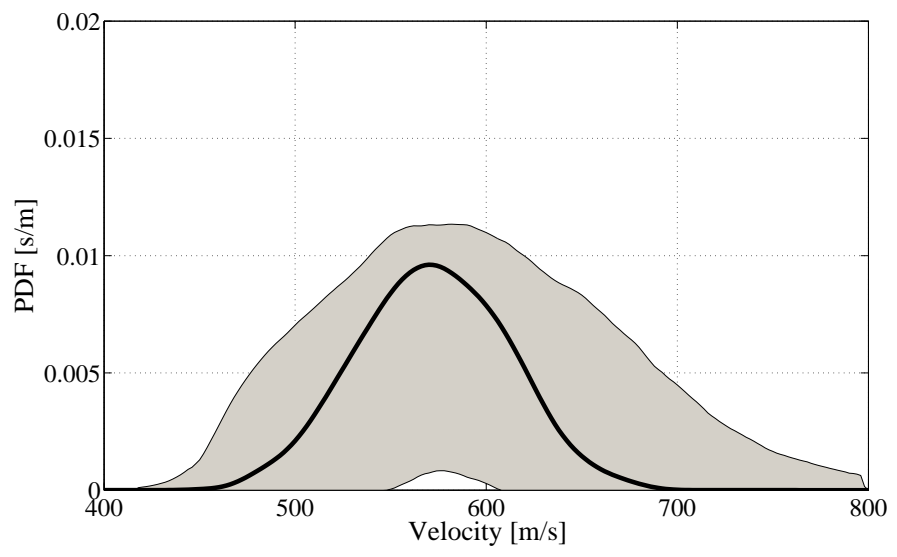

(a) $n=10$.

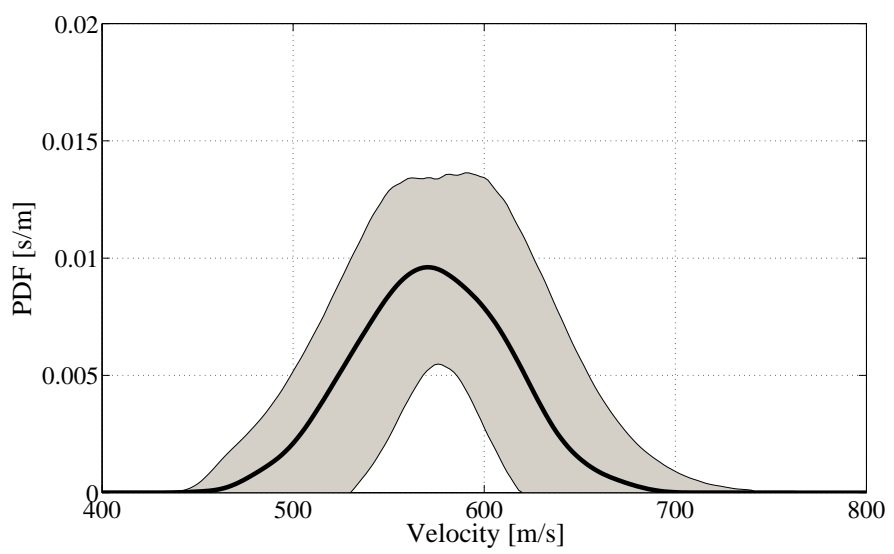

(b) $n=25$.

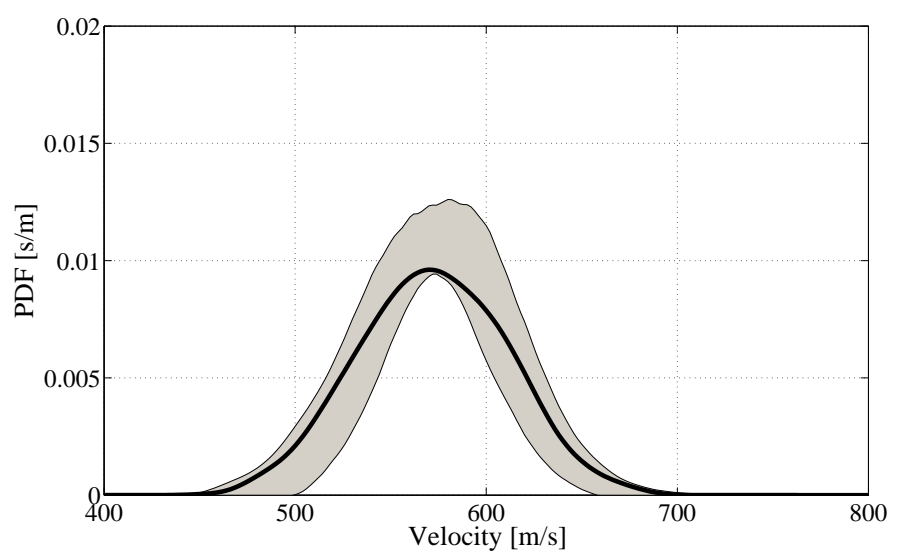

(c) $n=500$.

Fig. 12. Bayesian stochastic inversion: posterior 99\%-confidence region for the PDF for the onset of flutter for the data set of length (a) 10, (b) 25 and (c) 500 (filled), and ideal PDF predicted by the data-generating model (solid line). 


\section{List of Tables}

1 Simulated data: data set of length 10.

2 Maximum likelihood stochastic inversion: optimal coefficients identified from the data set of length 10 .

3 Bayesian stochastic inversion: 5 realizations of $\boldsymbol{p}^{0}$ and $\boldsymbol{p}^{\delta}$ obtained by MCMC sampling from the posterior associated with the data set of length 10 . 


\begin{tabular}{c|ccccc}
\hline$j[-]$ & $a_{1}^{(j)}[\mathrm{Hz}]$ & $a_{2}^{(j)}[\mathrm{Hz}]$ & $a_{3}^{(j)}[\mathrm{Hz}]$ & $a_{4}^{(j)}[\mathrm{Hz}]$ & $a_{5}^{(j)}[\mathrm{Hz}]$ \\
\hline 1 & 22.0644 & 38.7277 & 65.6377 & 102.7264 & 149.1156 \\
2 & 22.1918 & 39.3277 & 66.7944 & 104.3363 & 151.4071 \\
3 & 22.3315 & 38.9836 & 67.0343 & 103.7631 & 150.5413 \\
4 & 21.7841 & 38.1275 & 64.5681 & 100.6018 & 146.1258 \\
5 & 22.7409 & 40.4047 & 68.7806 & 107.0412 & 155.2916 \\
6 & 22.5119 & 40.0706 & 68.4612 & 106.8598 & 155.1919 \\
7 & 21.9770 & 38.6460 & 65.4526 & 102.1728 & 148.2193 \\
8 & 22.0674 & 38.7597 & 65.5012 & 102.6038 & 148.8000 \\
9 & 22.5796 & 39.5998 & 67.1365 & 105.1058 & 152.9256 \\
10 & 21.5810 & 37.6697 & 63.8471 & 99.9416 & 145.1024 \\
\hline
\end{tabular}

Table 1

Simulated data: data set of length 10 . 


\begin{tabular}{ccccccccc}
$\hat{p}_{1}^{0}$ & $\hat{p}_{2}^{0}$ & $\hat{p}_{3}^{0}$ & $\hat{p}_{4}^{0}$ & $\hat{p}_{5}^{0}$ & $\hat{p}_{101}^{\delta}$ & $\hat{p}_{102}^{\delta}$ & $\hat{p}_{011}^{\delta}$ & $\hat{p}_{012}^{\delta}$ \\
\hline 3.0993 & 3.6641 & 4.1937 & 4.6396 & 5.0126 & -0.9837 & -0.0209 & -0.0035 & -0.8734 \\
\hline
\end{tabular}

Table 2

Maximum likelihood stochastic inversion: optimal coefficients identified from the data set of length 10. 


\begin{tabular}{ccccccccc}
\hline$p_{1}^{0}$ & $p_{2}^{0}$ & $p_{3}^{0}$ & $p_{4}^{0}$ & $p_{5}^{0}$ & $p_{101}^{\delta}$ & $p_{102}^{\delta}$ & $p_{011}^{\delta}$ & $p_{012}^{\delta}$ \\
\hline 3.1005 & 3.6678 & 4.1879 & 4.6357 & 5.0029 & 0.2100 & -1.1100 & 1.9800 & 0.0300 \\
3.0977 & 3.6587 & 4.1819 & 4.6495 & 5.0087 & 0.1800 & 0.8400 & -0.6600 & 0.5400 \\
3.1056 & 3.6610 & 4.1949 & 4.6405 & 4.9979 & 0.2100 & 0.8700 & -1.5600 & 0.9600 \\
3.0977 & 3.6610 & 4.2001 & 4.6299 & 4.9954 & 0.9600 & -0.6900 & 0.4200 & 1.4400 \\
3.0926 & 3.6617 & 4.1819 & 4.6291 & 5.0162 & 0.9600 & -0.3600 & 0.2100 & -1.0800 \\
Table 3 & & & & & & & &
\end{tabular}

Bayesian stochastic inversion: 5 realizations of $\boldsymbol{p}^{0}$ and $\boldsymbol{p}^{\delta}$ obtained by MCMC sampling from the posterior associated with the data set of length 10 . 